\title{
TOPOLOGICAL EQUIVALENCE OF TILINGS
}

\author{
JOHANNES KELLENDONK \\ FACHBEREICH MATHEMATIK, TECHNISCHE UNIVERSITÄT BERLIN
}

\begin{abstract}
We introduce a notion of equivalence on tilings which is formulated in terms of their local structure. We compare it with the known concept of locally deriving one tiling from another and show that two tilings of finite type are topologically equivalent whenever their associated groupoids are isomorphic.
\end{abstract}

\section{INTRODUCTION}

In physics, tilings are used to model solids, in particular non-periodic ones. Studying the possible types of long range ordered structures (and their implication on physical quantities) amounts therefore in parts to the study of (a suitable class of) tilings. In fact, the investigation of certain tilings as idealized models for quasicrystals began more than a decade ago so that one can find by now a large amount of articles many of them being collected in SO87, JM95, AG95].

Some elements of a theory of long range ordered structures are based on tilings but require additional information, for instance when it comes to the calculation of diffraction patterns (Fourier transforms). Others depend only on the topological nature of the tiling, as e.g. the $K$-theoretical gap labelling. Results of these are consequently more qualitative in nature. The present article clearly belongs to the second area. In particular, the specific shape or volume of the tiles which make up the tiling will not be of importance for us. Furthermore, due to the locality of the interactions in the solid, it is only the local structure of the tiling that matters, i.e. the way the tiling looks on finite patches. One motivation to write this article is to illustrate that this local structure can be described by an almost-groupoid resp. an inverse semigroup. The groupoid associated to the tiling arises together with its topology functorially from the almost-groupoid. The algebraic structure is defined on the most elementary level and therefore underlies the construction of all topological invariants (including the group of possible gap labels) of the tiling.

The main aim of this article is however to give an answer to the question under which circumstances two tilings give rise to isomorphic groupoids. For that we introduce the notion of topological equivalence of tilings. This notion is closely related to mutually locally derivability of the tilings, a concept well known from physical considerations.

The article is organized as follows. We start with an informal description of the local structure of a tiling as an example of an almost-groupoid resp. inverse semigroup. After that we put this into a general context and describe a functor which assigns to every almost-groupoid a groupoid with discrete orbits. We apply this to tilings, obtaining the groupoid associated it, then emphasizing the particularities of this case.

Date: September 25, 1996.

This work was supported by the DFG. 
By that we mean the existence of a metric structure which is well known for tilings and with respect to which the functor looks like taking a closed subspace of the metric completion. We compare the groupoid, which we also call in distinction the discrete groupoid associated to the tiling, to the continuous groupoid, which is often considered in the literature.

In the next section we investigate the known concept of local derivability of tilings which leads us to introduce the notion of topological equivalence. Theorem 6 constitutes the main result of this article. It shows that topological equivalence of tilings - a purely "local" notion - is sufficient and necessary for them to have isomorphic groupoids. Whereas the metric structure is not used to define topological equivalence of tilings the proof of theorem relies on this structure. Everything is restricted to tilings which are of finite type. The finite type (or compactness) condition which they satisfy is the hypotheses for many compactness arguments.

In the final section we give a selected overview on topological invariants for tilings. We mention the invariants of the groupoid- $C^{*}$-algebra, the $K$-groups, and groupoid cohomology. But we will neither discuss the construction of groupoid- $C^{*}$-algebras (see [Ren80]) nor of its $K$-groups (see [Bla86]) nor of groupoid cohomology (see [Ren80, Kum88). We will also not illustrate the $K$-theoretical gap labelling but refer the reader to Bel92, BBG92, Kel95c].

\section{The LOCAL STRUCTURE OF A TILING}

In this article the following notion of tiling will be used. A tile (in $\mathbb{R}^{d}$ ) is a connected bounded subset of $\mathbb{R}^{d}$ which is the closure of its interior and may be decorated.] $\mathrm{A}$ $d$ dimensional tiling is an infinite set of tiles which cover $\mathbb{R}^{d}$ overlapping at most at their boundaries. A finite subset of a tiling is also called a pattern. Although often formulated for a specific tiling, the relevant quantities like the groupoid associated to it and the almost-groupoid of its local structure depend only on the congruence class of the tiling. A tile- resp. tiling- resp. pattern-class shall here be an equivalence class under translation of a tile resp. tiling resp. pattern, i.e. two such objects belong to the same class if there is an $x \in \mathbb{R}^{d}$ such that translation by $x$ applied to the tile resp. the elements of one set yield the other tile resp. the elements of the other. Note that a pattern class does not consist simply of tile classes.

The local structure of a tiling is a multiplicative structure determined by its pattern classes. On the set of patterns of a given tiling one can easily introduce an associative binary operation (multiplication), the union. But such an operation is not well defined on pattern classes. In order to achieve this we need to keep track of the relative position between patterns. This can be done with the help of an additional choice of a tile in the pattern, such a composed object is called a pointed pattern. Calling two pointed patterns composable if their choice of tile coincides (in the tiling), one may define an associative binary operation from the set of composable pairs into the set of pointed patterns as follows: the union of the patterns of the composable pair yields the new pattern and their common choice of tile the new choice. This multiplication being only partially defined, it appears at first sight to be a draw back, but its advantage lies in the possibility to extend it to a well defined partial multiplication on translation

1 The decoration, which may consist e.g. of arrows or colours, may serve for the purpose to distinguish translation classes of tiles which have the same shape. 
classes: Call two pointed pattern classes composable if they have representatives which are composable in the above sense, multiply them in case as above and take their translation class.

But this is not all we want. We want to be able to build arbitrary large pattern classes from a finite set of small ones using a multiplication. This can obviously not be achieved by the above. Instead, if we look at pattern classes with a choice of an ordered pair of tiles in it, calling that a doubly pointed pattern class, we can make larger pattern classes from smaller ones as follows: Ignoring the first resp. second choice in the ordered pair of the first resp. second pattern class we obtain two (simply) pointed pattern classes which may be multiplied as above provided they are composable. Of the resulting pointed pattern class we forget the choice of tile and take instead the ordered pair which is given by the so far ignored tiles, namely the first of the ordered pair of the first and the second of the ordered pair of the second pattern class we started with. As we will elaborate below, this is a useful algebraic structure which we call almost-groupoid. Equivalently one could work, after adding a zero element, with inverse semigroups [Pet84]. We still keep the name almost-groupoid, because it is almost a groupoid and applying a functor to it yields topological groupoids. This functor is most natural in tiling theory since it furnishes tilings from patterns.

1.1. Almost-groupoids / inverse semigroups. Let $\Gamma$ be a set. A partially defined associative multiplication is given by a subset $\Gamma^{\vdash} \subset \Gamma \times \Gamma$ of composable pairs (we write $x \vdash y$ for $(x, y) \in \Gamma^{\vdash}$ ) with a map $m: \Gamma^{\vdash} \rightarrow \Gamma$ (we write $x y=m(x, y)$ ) which is associative in the sense that, first, $x \vdash y$ and $x y \vdash z$ is equivalent to $x \vdash y z$ and $y \vdash z$, and second, if $x \vdash y$ and $x y \vdash z$ then $(x y) z=x(y z)$. Hence we don't have to care about brackets.

Relations or equations like the above in a set with partially defined associative multiplication make sense only if the multiplications are defined, i.e. if the to be multiplied pairs are composable. In order to avoid cumbersome notation we shall agree from now on that a relation involving products is true if all multiplications involved are defined and it is then true.

Given such a set $\Gamma$ with partially defined multiplication, suppose that for some $a \in \Gamma$ the equations $a x a=a$ and $x a x=x$ were true for some $x \in \Gamma$. Then $x$ is called an inverse of $a$. $\Gamma$ has a unique inverse (map) if any $a$ has a unique inverse. The inverse map is then denoted by $a \mapsto a^{-1}$.

Definition 1. An almost-groupoid is a set $\Gamma$ with partially defined associative multiplication and unique inverse.

A set with fully defined associative multiplication and unique inverse is an inverse semigroup, i.e. an inverse semigroup is an almost-groupoid for which $\Gamma^{\vdash}=\Gamma \times \Gamma$. In particular, adding a zero element 0 to an almost groupoid $\Gamma$ and extending the multiplication by $x y=0$ if $x \forall y$, and $0 x=x 0=0$, yields an inverse semigroup with zero (which we write as $\Gamma_{0}$ ). Conversely, if $\Gamma_{0}$ is an inverse semigroup with zero then $\Gamma=\Gamma_{0} \backslash\{0\}$ with $\Gamma^{\vdash}=\{(x, y) \mid x y \neq 0\}$ is an almost-groupoid. So we may apply the known results of inverse semigroup theory. In fact, any statement below on almostgroupoids may be reformulated as a statement on inverse semigroups with zero element and vice versa. However, we find the formulation in terms of almost-groupoids more natural. 
The elements of $\Gamma^{0}:=\left\{x x^{-1} \mid x \in \Gamma\right\}$ are called units. There are the image of the frequently occurring maps $r, d: \Gamma \rightarrow \Gamma^{0}$ given by $r(x)=x x^{-1}$ and $d(x)=r\left(x^{-1}\right)$ Let us mention that the uniqueness of the inverse implies, first, that units are the same as idempotents, i.e. $\Gamma^{0}:=\left\{x \in \Gamma \mid x^{2}=x\right\}$, and that they commute, and second, that the inverse map is an involution, in particular $(x y)^{-1}=y^{-1} x^{-1}$. A proof of that can be found in [Pet84] formulated in the framework of inverse semigroups.

This has implications on which kind of elements are composable. E.g. if $x \vdash y$ then $(x y)^{-1}=y^{-1} x^{-1}$ so that we must also have $y^{-1} \vdash x^{-1}$. Furthermore, under the same condition $x \vdash y$ we have $x y=x y y^{-1} x^{-1} x y$ so that we must have composabilities like $x y \vdash y^{-1}$ etc.. Similarly, $d(x)=r(y)$ implies $x=x y y^{-1}$ so that we must have $x \vdash y$. If $x \vdash y$ is even equivalent to $d(x)=r(y)$, then $\Gamma$ satisfies cancellation, i.e. $x y=x z$ implies $y=z$. This is simply because for $x y=x z$ to be true we must have $x \vdash y$ and $x \vdash z$. But then $y=r(y) y=d(x) y=d(x) z=z$.

Note that a groupoid - for an explicit definition c.f. Ren80 - is the same as an almost-groupoid which satisfies cancellation.

The well known order relation on inverse semigroups [Pet84 will be of great use here. One way of formulating it here is:

Definition 2. The order of an almost-groupoid is defined by

$$
x \preceq y \quad \text { whenever } r(x)=x y^{-1} \text {. }
$$

Note that $x \succeq y$ is equivalent to $x^{-1} \succeq y^{-1}$, and, if moreover $y \vdash z$, then $x \vdash z$ and $x z \succeq y z$. In other words the order is compatible with multiplication. Note also that a groupoid has trivial order.

Lemma 1. The set of all minimal elements of an almost-groupoid is a (possibly empty) ideal which is a groupoid.

Proof: Let $\Gamma$ be an almost-groupoid and $x \vdash y$ for two of its elements. Suppose that $x$ is minimal and consider the relation $x y \succeq z$, i.e. $z^{-1} z=z^{-1} x y$. We want to show that $z=x y$ and hence it is minimal. Since order is compatible with multiplication we have $x \succeq z y^{-1}$ hence $x=z y^{-1}$ by minimality. Since for units $u$ holds $z u \preceq z$ we conclude $x x^{-1}=z y^{-1} y z^{-1} \preceq z z^{-1}$, and $x x^{-1} x y \preceq z z^{-1} x y=z$ showing that $x y=z$. Thus $x y$ is minimal. In particular, all minimal elements form an almost-groupoid (which may be empty). We want to show that it satisfies cancellation, i.e. that $x \vdash y$ implies $d(x)=r(y)$. If $x \vdash y$ then $d(x) \vdash r(y)$ and hence $d(x), r(y) \succeq d(x) r(y)$. Minimality of $x$ implying that of $d(x)$ and $r(x)$ shows that $d(x)=r(y)$.

q.e.d.

Let $u \in \Gamma^{0}$ and $c \in \Gamma$. If $c \preceq u$ then $c^{-1}=d(c) u$ and in particular $c \in \Gamma^{0}$. On the other hand $u \preceq c$ does for $u \in \Gamma^{0}$ not have to imply that $c \in \Gamma^{0}$. But this latter property is useful in the sequel so that we give it a name.

Definition 3. An almost-groupoid is unit hereditary if, for $u \in \Gamma^{0}$ and $c \in \Gamma, u \preceq c$ implies $c \in \Gamma^{0}$.

Either of the statements $x y^{-1} \in \Gamma^{0}$ or $y x^{-1} \in \Gamma^{0}$ implies that $x$ and $y$ have a lower bound (common smaller element). E.g. if $x y^{-1} \in \Gamma^{0}$ then $x d(y)$ is such a lower bound.

\footnotetext{
${ }^{2}$ We use here a direction of the order which coincides with the convention used in semigroup theory. It is reversed to that in Kel95b.
} 
For a unit hereditary almost-groupoid the converse holds as well, namely if $x$ and $y$ have a lower bound $z$ then $r(z)$ is smaller than both, $x y^{-1}$ and $y x^{-1}$. Moreover, in that case $z=z d(z) \preceq x d(y)$. Therefore, if $\Gamma$ is unit hereditary and $x$ and $y$ have a lower bound then

$$
\max \{z \in \Gamma \mid z \preceq x, y\}=x d(y)
$$

and $r(x) y=r(y) x=y d(x)=x d(y)$.

Example 1. Let $X$ be a topological space and $\beta_{0}(X)$ a (not necessarily proper) subset of the topology of $X$ which has the property that any open subset of $X$ is a union of sets of $\beta_{0}(X)$ (i.e. it is a base of the topology) and that it is closed under intersection. Then $U V=U \cap V$ defines a multiplication on $\beta_{0}(X)$. Since the only solution of the equations $U \cap V \cap U=U$ and $V \cap U \cap V=V$ is given by $U=V$ and $U \cap U=U, \beta_{0}(X)$ is a commutative inverse semigroup which consists of units (idempotents) only. The empty set is a zero element in it and consequently $\beta(X):=\beta_{0}(X) \backslash\{\emptyset\}$ a commutative almost-groupoid consisting of units only. Its order is the inclusion of sets. Note that there are in general no minimal elements in $\beta(X)$.

Example 2. Let $\mathcal{T}$ be a tiling of $\mathbb{R}^{d}$. We already have explained in words that the set $\mathcal{M}_{\text {II }}$ of doubly pointed pattern classes carries a partially defined multiplication. Let us reformulate this in more technical terms. We start with defining an order relation on $\mathcal{M}_{\mathrm{II}}$, namely $c \succeq c^{\prime}$ if $c^{\prime}$ can be obtained from $c$ by addition of tiles but keeping the ordered pair of chosen tiles fixed. Let $\mathcal{M}_{\mathrm{II}}$ be the set of all pattern classes together with an ordered triple of chosen tiles and denote for $\eta \in \mathcal{M}_{\mathrm{II}}$ by $\eta_{\hat{i}} \in \mathcal{M}_{\mathbb{I I}}$ the doubly pointed pattern class which is obtained by forgetting the $i$ th choice in the triple. Call two doubly pointed pattern classes $c, c^{\prime}$ composable whenever there is an $\eta \in \mathcal{M}_{\text {III }}$ such that $c \succeq \eta_{\hat{3}}$ and $c^{\prime} \succeq \eta_{\hat{1}}$. Then define the product of two composable elements

$$
c c^{\prime}=\max \left\{\eta_{\hat{2}} \mid \eta \in \mathcal{M}_{\mathrm{III}}, c \succeq \eta_{\hat{3}}, c^{\prime} \succeq \eta_{\hat{1}}\right\}
$$

the maximum being taken with respect to the above order. This defines an associative multiplication. It turns out to have a unique inverse map $c \mapsto c^{-1}$ which is given by interchange of the elements of the ordered pair of chosen tiles. Thus $\mathcal{M}_{\mathbb{I}}$ forms an almost-groupoid which is in general not commutative. The order of the almostgroupoid coincides with the order used to define composability. In particular, the almost-groupoid of a tiling is unit hereditary. Note that there are no minimal elements in $\mathcal{M}_{\mathrm{II}}$.

A well known equivalence relation among tilings is that of two tilings being locally isomorphic [SS86]. Thus are called two tilings which have the property that every pattern class of either tiling can also be found in the other. This can here simply be expressed by saying that the tilings lead to the same almost-groupoid.

Let $\mathcal{M}_{\mathrm{I}}$ be the set of pointed pattern classes which are pattern classes together with one chosen tile. We may identify $\mathcal{M}_{\mathrm{I}}$ with the subset of $\mathcal{M}_{\mathbb{I}}$ consisting of those elements which are invariant under the inverse map, i.e. for which the chosen tiles in the ordered pair coincide. Another specific property which holds for almost-groupoids defined by

${ }^{3}$ The notion is used here in a stronger sense than in [SS86] in that pattern classes are considered as equivalence classes under translations but not under rotations. 
tilings is that elements which are equal to their inverse have to be units, i.e. under the above identification $\mathcal{M}_{\mathrm{I}}=\mathcal{M}_{\mathrm{II}}^{0}$.

We shall be interested in tilings which satisfy the following finite type (or compactness) condition. We call a pattern (and its class) connected if the subset it covers is connected.

- The set of connected doubly pointed pattern classes which consist of two tiles is finite.

Since tiles are bounded sets which have positive Lebesgue measure this condition implies that, for any $r$, the maximal number of tiles a pattern fitting inside an $r$-ball can have is finite. From that one concludes that the above condition is equivalent to the requirement that the number of pattern classes fitting inside an $r$-ball is finite. In particular $\mathcal{M}_{\mathbb{I}}$ is countable.

1.2. From almost-groupoids to groupoids. We now aim at a functorial construction to obtain a topological groupoid from an almost-groupoid. For that we consider sequences $\left(x_{n}\right)_{n \in \mathbb{N}}$ of elements $x_{n} \in \Gamma$ which are decreasing in that for all $n: x_{n} \succeq x_{n+1}$. The set of all decreasing sequences, which is denoted by $\Gamma_{\succeq}^{\mathbb{N}}$, carries a pre-order

$$
\left(x_{n}\right)_{n} \preceq\left(y_{n}\right)_{n} \quad \text { whenever } \forall n \exists m: x_{m} \preceq y_{n} .
$$

To turn this pre-order into an order one considers the equivalence relation on $\Gamma_{\succeq}^{\mathbb{N}}$

$$
\left(x_{n}\right)_{n} \sim\left(y_{n}\right)_{n} \text { whenever }\left(x_{n}\right)_{n} \preceq\left(y_{n}\right)_{n} \text { and }\left(y_{n}\right)_{n} \preceq\left(x_{n}\right)_{n} .
$$

On the set of equivalence classes, the elements of which we denote by $\left[\left(x_{n}\right)_{n}\right]$,

$$
\left[\left(x_{n}\right)_{n}\right] \preceq\left[\left(y_{n}\right)_{n}\right] \quad \text { whenever } \quad\left(x_{n}\right)_{n} \preceq\left(y_{n}\right)_{n}
$$

is an order relation.

Definition 4. For a given almost-groupoid $\Gamma, \widetilde{\Gamma}$ is the set $\Gamma_{\succeq}^{\mathbb{N}}$ modulo relation (因) and $\mathcal{R}(\Gamma)$ the set of minimal elements of $\widetilde{\Gamma}$ with respect to the order (5).

We identify the elements of $\Gamma$ with constant sequences in $\widetilde{\Gamma}$. We use also the notation $\widetilde{x}$ for the elements of $\widetilde{\Gamma}$.

Lemma 2. If $\Gamma$ is a countable almost-groupoid then any $x \in \Gamma$ has a smaller minimal element in $\widetilde{\Gamma}$, in particular $\mathcal{R}(\Gamma) \neq \emptyset$.

Proof: Given $x \in \Gamma$ there is a bijection $\gamma: \mathbb{N} \rightarrow \Gamma$ such that $\gamma(1)=x$. Now define $\hat{\gamma}(1)=\hat{\gamma}(1)$ and $\hat{\gamma}(n)=\hat{\gamma}(n-1) d(\gamma(n))$ if $\hat{\gamma}(n-1) \vdash d(\gamma(n))$ and else $\hat{\gamma}(n)=\hat{\gamma}(n-1)$. Then $(\hat{\gamma}(n))_{n} \in \Gamma_{\succeq}^{\mathbb{N}}$. Now suppose that $\left(y_{n}\right)_{n} \preceq(\hat{\gamma}(n))_{n}$. Then in particular $y_{m}$ and $\hat{\gamma}(n)$ have for all $n, m \in \mathbb{N}$ a common smaller element. But this implies that $\hat{\gamma}\left(\gamma^{-1}\left(y_{m}\right)\right) \preceq y_{m}$ and hence $\left(y_{n}\right)_{n} \succeq(\hat{\gamma}(n))_{n}$. Thus $(\hat{\gamma}(n))_{n}$, which is certainly smaller than the constant sequence $x$, is a minimal element.

q.e.d.

Examples show that countability is not a necessary condition.

Lemma 3. $\widetilde{\Gamma}$ is an almost-groupoid under the operations induced by point-wise operations on $\Gamma_{\succeq}^{\mathbb{N}}$, and its order coincides with the order (5). 
Proof: $\Gamma_{\succeq}^{\mathbb{N}}$ is an almost-groupoid under point-wise operations, i.e. composability is given by $\left(x_{n}\right)_{n} \vdash\left(y_{n}\right)_{n}$ if $\forall n: x_{n} \vdash y_{n}$ and then $\left(x_{n}\right)_{n}\left(y_{n}\right)_{n}=\left(x_{n} y_{n}\right)_{n},\left(x_{n}\right)_{n}^{-1}=\left(x_{n}^{-1}\right)_{n}$. Since order is compatible with multiplication $\left(x_{n}^{\prime}\right)_{n} \sim\left(x_{n}\right)_{n}$ and $\left(y_{n}^{\prime}\right)_{n} \sim\left(y_{n}\right)_{n}$ and $\left(x_{n}\right)_{n} \vdash\left(y_{n}\right)_{n}$ imply, first $\left(x_{n}^{\prime}\right)_{n} \vdash\left(y_{n}^{\prime}\right)_{n}$, and second $\left(x_{n} y_{n}\right)_{n} \sim\left(x_{n}^{\prime} y_{n}^{\prime}\right)_{n}$. Furthermore $x \succeq y$ being equivalent to $x^{-1} \succeq y^{-1}$ implies that $\left(x_{n}\right)_{n} \sim\left(y_{n}\right)_{n}$ is equivalent to $\left(x_{n}^{-1}\right)_{n} \sim\left(y_{n}^{-1}\right)_{n}$. From this follows the uniqueness of inversion. Hence also $\widetilde{\Gamma}$ is an almost-groupoid. Its units are classes of sequences of decreasing units of $\Gamma$. It is straightforward to see that its order is given by (5). q.e.d.

1.2.1. Morphisms of almost-groupoids. It turns out that the natural morphisms to look at in the context of tilings are not homomorphisms but certain prehomomorphisms. An order ideal of an almost-groupoid $\Gamma$ is a subset $\mathcal{N}$ for which $c \preceq c^{\prime} \in \mathcal{N}$ implies $c \in \mathcal{N}$. Any subset $\mathcal{N}$ generates an order ideal, namely $I(\mathcal{N})=\{x \in \Gamma \mid \exists y \in \mathcal{N}: x \preceq y\}$. We call an element of $\Gamma_{\succeq}^{\mathbb{N}}$ approximating if its class is minimal.

Definition 5. A prehomomorphism $\varphi: \Gamma \rightarrow \Gamma^{\prime}$ between two almost-groupoids is a map which preserves composability, commutes with the inversion map, and satisfies for all $x \vdash y$

$$
\varphi(x y) \preceq \varphi(x) \varphi(y) .
$$

A prehomomorphism is called approximating if it maps approximating sequences onto approximating ones. An approximating prehomomorphism $\varphi: D(\varphi) \subset \Gamma \rightarrow \Gamma^{\prime}$ is called a partial approximating prehomomorphism or local morphism between $\Gamma$ and $\Gamma^{\prime}$ if its domain $D(\varphi)$, which is a sub-almost-groupoid of $\Gamma$, is an order ideal.

Lemma 4. Prehomomorphisms preserve the order.

Proof: $x \preceq y$ is equivalent to $x=r(x) y$ and hence implies $\varphi(x) \preceq r(\varphi(x)) \varphi(y) \preceq$ $\varphi(y)$. q.e.d.

This lemma implies that prehomomorphisms are composable, and since the domain of $\psi \circ \varphi$, which is $D(\psi \circ \varphi)=\{x \in D(\varphi) \mid \varphi(x) \in D(\psi)\}$, is an order ideal of $\Gamma$ local morphisms are composable as well. A prehomomorphism $\varphi: \Gamma \rightarrow \Gamma^{\prime}$ of almostgroupoids can be extended to a prehomomorphism $\varphi: \Gamma_{0} \rightarrow \Gamma_{0}^{\prime}$ of inverse semigroups by simply setting $\varphi(0)=0$. The condition that $\varphi: \Gamma \rightarrow \Gamma^{\prime}$ preserves composability implies then for the extension that it satisfies $\varphi^{-1}(0)=0$. Conversely, any prehomomorphism $\varphi: \Gamma_{0} \rightarrow \Gamma_{0}^{\prime}$ of inverse semigroups with zero which satisfies $\varphi^{-1}(0)=0$ restricts to a prehomomorphism on the almost-groupoids. A homomorphisms between almostgroupoids is a prehomomorphism for which (6) is an equality.

By element wise application to sequences, a prehomomorphism maps decreasing sequences onto decreasing sequences, and moreover preserves equivalence classes. Hence it extends to a prehomomorphism $\tilde{\varphi}: \widetilde{\Gamma} \rightarrow \widetilde{\Gamma}^{\prime}$ through

$$
\widetilde{\varphi}\left[\left(x_{n}\right)_{n}\right]:=\left[\left(\varphi\left(x_{n}\right)\right)_{n}\right] .
$$

If $\varphi$ is a local morphism then we denote by $\mathcal{R}(\varphi): \mathcal{R}(D(\varphi)) \rightarrow \mathcal{R}(\Gamma)$ the restriction of $\widetilde{\varphi}$ to the minimal elements $\mathcal{R}(D(\varphi))$. 
1.2.2. Topology. A topological almost-groupoid is an almost-groupoid which carries a topology such that the product and the inversion map are continuous, $\Gamma^{\vdash}$ carrying the relative topology. A (locally compact) groupoid is called $r$-discrete if $r^{-1}(x)$ is discrete for any $x$, or equivalently, if its set of units $\Gamma^{0}$ is open Ren80.

If nothing else is said $\Gamma$ shall carry the discrete topology. The topology of $\widetilde{\Gamma}$ shall then be defined as the one which is generated by $\beta_{0}(\widetilde{\Gamma}):=\left\{\widetilde{U}_{x} \mid x \in \Gamma_{0}\right\}$,

$$
\widetilde{U}_{x}=\{\widetilde{y} \in \widetilde{\Gamma} \mid \widetilde{y} \preceq x\},
$$

$\widetilde{U}_{0}=\emptyset$, and $\mathcal{R}(\Gamma)$ shall carry the relative topology, i.e. the one generated by $\beta_{0}(\mathcal{R}(\Gamma))=$ $\left\{\mathcal{U}_{x}, x \in \Gamma\right\}, \mathcal{U}_{x}=\widetilde{U}_{x} \cap \mathcal{R}(\Gamma)$. Using set multiplication $\|^{\prime}$ as multiplication on $\beta_{0}(\widetilde{\Gamma})$ resp. $\beta_{0}(\mathcal{R}(\Gamma))$ we get:

Lemma 5. The maps $x \mapsto \widetilde{U}_{x}$ resp. $x \mapsto \mathcal{U}_{x}$ furnish an isomorphisms between the inverse semigroups $\Gamma_{0}$ and $\beta_{0}(\widetilde{\Gamma})$ resp. $\beta_{0}(\mathcal{R}(\Gamma))$.

Proof: Let $x, y \in \Gamma . \widetilde{U}_{x} \widetilde{U}_{y} \subset \widetilde{U}_{x y}$ follows directly from the compatibility between order and multiplication and $\mathcal{U}_{x} \mathcal{U}_{y} \subset \mathcal{U}_{x y}$ is then a consequence of Lemma 1. As for the converse, let $\widetilde{z} \preceq x y$. Then first $x^{-1} \widetilde{z} \preceq x^{-1} x y \preceq y$, and second $\widetilde{z}=r(x y) \widetilde{z} \preceq r(x) \widetilde{z}$ hence $\widetilde{z}=x\left(x^{-1} \widetilde{z}\right)$. This shows that $\widetilde{z} \in \widetilde{U}_{x} \widetilde{U}_{y}$. If moreover $\widetilde{z}$ is minimal then the factorization $\widetilde{z}=(r(\widetilde{z} x))\left(x^{-1} \widetilde{z}\right)$ shows $\widetilde{z} \in \mathcal{U}_{x} \mathcal{U}_{y}$ as both, $r(\widetilde{z} x)$ and $x^{-1} \widetilde{z}$ are minimal. Thus

$$
\widetilde{U}_{x} \widetilde{U}_{y}=\widetilde{U}_{x y} \text { and } \mathcal{U}_{x} \mathcal{U}_{y}=\mathcal{U}_{x y} .
$$

The considered maps are by definition surjective. But either of $\widetilde{U}_{x}=\widetilde{U}_{y}$ or $\mathcal{U}_{x}=\mathcal{U}_{y}$ implies that $x \preceq y$ and $y \preceq x$ so that the maps are injective as well.

q.e.d.

If $\Gamma$ is unit hereditary $\beta_{0}(\widetilde{\Gamma})$ and $\beta_{0}(\mathcal{R}(\Gamma))$ are closed under intersection. In fact, $\widetilde{U}_{x} \cap \widetilde{U}_{y} \neq \emptyset$ whenever $x$ and $y$ have a lower bound in $\Gamma$ and therefore $\widetilde{U}_{x} \cap \widetilde{U}_{y}=\widetilde{U}_{r(x) y}$ (which might be empty) and hence also $\mathcal{U}_{x} \cap \mathcal{U}_{y}=\mathcal{U}_{r(x) y}$.

Theorem 1. $\mathcal{R}(\Gamma)$ is an r-discrete topological groupoid whose topology is $T_{1}$. If $\Gamma$ is unit hereditary then $\mathcal{R}(\Gamma)$ is even Hausdorff.

Proof: $\mathcal{U}_{x}^{-1}=\mathcal{U}_{x^{-1}}$ is open showing continuity of the inversion. By (9),

$$
m^{-1}\left(\mathcal{U}_{x}\right)=\bigcup_{\left(x_{1}, x_{2}\right) \in \Gamma^{\vdash}: x \succeq x_{1} x_{2}}\left(\mathcal{U}_{x_{1}} \times \mathcal{U}_{x_{2}}\right) \cap \mathcal{R}(\Gamma)^{\vdash}
$$

is open as well and hence multiplication continuous.

Moreover, since $d\left(\left[\left(x_{n}\right)_{n}\right]\right) \preceq d\left(x_{1}\right)$ we have $\mathcal{R}(\Gamma)^{0}=\bigcup_{u \in \Gamma^{0}} \mathcal{U}_{u}$ which is open and hence the groupoid $r$-discrete.

To show that $\mathcal{R}(\Gamma)$ is $T_{1}$, i.e. that for all $\widetilde{x}, \widetilde{y} \in \mathcal{R}(\Gamma)$ with $\widetilde{x} \neq \widetilde{y}$ there is an open $U$ containing $\widetilde{x}$ but not $\widetilde{y}$, let $\left(x_{n}\right)_{n}$ resp. $\left(y_{n}\right)_{n}$ be a representative for $\widetilde{x}$ resp. $\widetilde{y}$ observe that $\widetilde{x} \neq \widetilde{y}$ implies both, $\widetilde{x} \npreceq \widetilde{y}$ and $\widetilde{x} \nsucceq \widetilde{y}$, and hence the existence of an $n_{0}$ such that for all $n \geq n_{0}: x_{n} \nsucceq \widetilde{y}$ and $y_{n} \nsucceq \widetilde{x}$. Therefore any $U=\mathcal{U}_{x_{n}}, n \geq n_{0}$, does the job.

Now suppose that $\Gamma$ is unit hereditary. We claim that for some $m, x=x_{n_{0}}$ and $y_{m}$ do not have a smaller common element. This then proves the Hausdorff property since for that $m$ is $\mathcal{U}_{x} \cap \mathcal{U}_{y_{m}}=\emptyset$ and $\widetilde{x} \in \mathcal{U}_{x}$ and $\widetilde{y} \in \mathcal{U}_{y_{m}}$. To prove the claim suppose its

\footnotetext{
${ }^{4}$ For arbitrary subsets of $\widetilde{\Gamma}_{0}$ is $\widetilde{U} \widetilde{V}=\{\widetilde{x} \widetilde{y} \mid \widetilde{x} \in \widetilde{U}, \widetilde{y} \in \widetilde{V}, \widetilde{x} \vdash \widetilde{y}\}$.
} 
contrary, i.e. $x$ and $y_{m}$ to have a common smaller element for all $m$. Then $\widetilde{y} d(x) \preceq \widetilde{y}$ which by minimality implies $\widetilde{y} \preceq \widetilde{y} d(x)$. In particular $\exists l \exists m: y_{m} \preceq y_{l} d(x)$, and since $y_{l} x^{-1}$ is a unit $y_{l} d(x) \preceq x$. This contradicts the above.

q.e.d.

Theorem 2. Let $\varphi: D(\varphi) \rightarrow \Gamma^{\prime}$ be an local morphism of almost-groupoids and $\mathcal{R}(\varphi)$ be the restriction of $\tilde{\varphi}$ to $\mathcal{R}(D(\varphi))$. Then $\mathcal{R}(\varphi): \mathcal{R}(D(\varphi)) \rightarrow \mathcal{R}\left(\Gamma^{\prime}\right)$ is a continuous homomorphism between topological groupoids.

Proof: $\mathcal{R}(\varphi)$ is a prehomomorphism by construction. But cancellation implies that on groupoids the order is trivial and hence prehomomorphisms are homomorphisms. To show continuity of $\mathcal{R}(\varphi)$ let $x^{\prime} \in \Gamma^{\prime}$. Then $\mathcal{R}(\varphi)\left(\left[\left(x_{n}\right)_{n}\right]\right) \in \mathcal{U}_{x^{\prime}}$ is equivalent to $\exists n: x^{\prime} \succeq \varphi\left(x_{n}\right)$. Hence $\mathcal{R}(\varphi)^{-1}\left(\mathcal{U}_{x^{\prime}}\right) \subset \bigcup_{y \in \Gamma: \varphi(y) \preceq x^{\prime}} \mathcal{U}_{y}$. Since also $\mathcal{R}(\varphi)\left(\mathcal{U}_{y}\right) \subset \mathcal{U}_{\varphi(y)}$, and $x \preceq y$ implies $\mathcal{U}_{x} \subset \mathcal{U}_{y}$, the above inclusion is in fact an equality. This shows continuity.

q.e.d.

In fact, it is easily checked that $\mathcal{R}$ is a covariant functor of the category of almostgroupoids with local morphisms into the category of $r$-discrete groupoids with partial continuous homomorphisms. Since $\mathcal{R}(\Gamma)$ has trivial order decreasing sequences are constant sequences. Therefore we may identify $\mathcal{R} \circ \mathcal{R}$ with $\mathcal{R}$.

In general an almost-groupoid is non commutative. But in Example 1 we have seen that bases of the topology of topological spaces which are closed under intersection give rise to almost-groupoids which consist only of units so they are in particular commutative. Theorem 1 and (9) show that any almost-groupoid may be identified (after adding a zero element) with a base of the topology of a $T_{1}$ space but only in the case where the almost-groupoid consists only of units its multiplication coincides with intersection. In that case $\mathcal{R}(\Gamma)=\mathcal{R}(\Gamma)^{0}$. Hence if $x \vdash y$, which means for groupoids $d(x)=r(y)$, then $y=x$. In other words the groupoid operations are trivial, i.e. $x$ is composable only with itself, $x^{2}=x$, and $x^{-1}=x$. So a topological groupoid which consists only of units is an ordinary topological space. This indicates why one may call the field to which this study of tilings belongs the non commutative topology of tilings.

1.2.3. Inverse semigroups of groupoids. It is instructive to compare the inverse semigroup $\Gamma_{0}$ from which we obtained the groupoid with other inverse semigroups which are often considered in connection with groupoids. For instance in Renault's book Ren80 such inverse semigroups (with zero) are considered which consist of $G$-sets. A $G$-set is a subset $s$ of the groupoid $G$ which has the property that the restrictions of $r$ and $d$ to $s$ are both injective. Multiplication is then given by set multiplication (and inversion applies element-wise). The order is inclusion of sets and the empty set is the zero element. If no more restrictions on the $G$-sets are given this is called the inverse semigroup of the groupoid, we denote it here by $\mathcal{I S G}(G)$. In the context of $r$-discrete groupoids it is also interesting to look at those $G$-sets which are compact and open. They form also a sub-inverse semigroup, the ample semigroup of $G$ denoted here by $\mathcal{A S G}(G)$. Note that both, $\mathcal{I S G}(G)$ and $\mathcal{A S G}(G)$ are closed under intersection. Since the assignment of an inverse semigroup to the groupoid is reverse to the functor $\mathcal{R}$ the natural question is whether they are somehow inverse (leaving aside the more subtle question of how to assign to a groupoid homomorphism a morphism of $\mathcal{I S G}(G)$ or $\mathcal{A S G}(G))$. The answer is in general negative but we can say the following.

The relation between the inverse semigroup $\Gamma_{0}$ to start with and the inverse semigroups of $\mathcal{R}(\Gamma)$-sets of $\mathcal{R}(\Gamma)$ is rather obvious: $\mathcal{U}_{c}, c \in \Gamma$, is a $\mathcal{R}(\Gamma)$-set so that by $(\mathbf{9})$ 
we may identify $\Gamma_{0}$ as a sub-inverse semigroup of $\mathcal{I S G}(\mathcal{R}(\Gamma))$. Furthermore, if the $\mathcal{U}_{c}$ are compact then $\mathcal{A S G}(\mathcal{R}(\Gamma))$ is given by (finite) unions of elements of $\Gamma_{0}$ under this identification. But they are not equal (and not all finite unions are allowed).

A topological space is called first countable if any point has a countable local base. If it is $T_{1}$ then for any point $x$ there exists a descending sequence $\left(U_{n}\right)_{n}$ of neighbourhoods such that $\bigcap_{n} U_{n}=\{x\}$. Such a sequence may be constructed as follows: Let $\mathcal{U}(x)$ be a local base at $x$ and $\gamma: \mathbb{N} \rightarrow \mathcal{U}(x)$ be a bijection. Define $\hat{\gamma}(1)=\gamma(1)$ and $\hat{\gamma}(n)=\gamma(m)$ where $m$ is the smallest number such that $\gamma(m) \subset \gamma(n) \cap \hat{\gamma}(n-1)$. This is a descending sequence and $x \in \bigcap_{n} \hat{\gamma}(n)$. Let $y \neq x$ and $V$ be an open set containing $x$ but not $y$. Then there is a $U \in \mathcal{U}(x)$ such that $U \subset V$ and hence $y \notin \hat{\gamma}\left(\gamma^{-1}(U)\right)$. Hence $y \notin \bigcap_{n} \hat{\gamma}(n)$. Thus the $\hat{\gamma}(n)$ form the desired sequence.

Theorem 3. If $G$ is a first countable groupoid whose topology is $T_{1}$ and generated by $\mathcal{A S G}(G)$ then $\mathcal{R}(\mathcal{A S G}(G) \backslash\{\emptyset\})=G$.

Proof: Let $\Gamma=\mathcal{A S G}(G) \backslash\{\emptyset\}$. The map $p\left(\left[\left(U_{n}\right)_{n}\right]=\bigcap_{n} U_{n}\right.$ is easily seen to be a well defined map from $\widetilde{\Gamma}$ into the power set of $G$. Since the elements of $\mathcal{A S G}(G)$ are compact $p\left(\left[\left(U_{n}\right)_{n}\right]\right)$ is not empty. Now suppose that $\left[\left(U_{n}\right)_{n}\right]$ were minimal and $x \neq y$ both in $\bigcap_{n} U_{n}$. Then there is a $V \in \mathcal{A S G}(G): y \notin V, x \in V$. It follows that $\left[\left(V \cap U_{n}\right)_{n}\right]$ is strictly smaller than $\left[\left(U_{n}\right)_{n}\right]$ which yields a contradiction. We conclude that $p$ maps $\mathcal{R}(\beta(X))$ onto singletons. Hence $p$ defines a map $p^{\prime}: \mathcal{R}(\beta(X)) \rightarrow X$.

Since $G$ is first countable and $T_{1}$ any point $x$ lies in the image of $p^{\prime}$, namely according to the above remark we can find a descending sequence of neighborhoods $(\hat{\gamma}(n))_{n}$ with $\{x\}=\bigcap_{n} \hat{\gamma}(n)$. Now choose for any $n$ an $U_{n}^{\prime} \in \mathcal{A S G}(G)$ with $x \in U_{n}^{\prime} \subset \hat{\gamma}(n)$, and set $U_{n}=\bigcap_{i<n} U_{i}^{\prime}$. Then $\widetilde{U}$ is a pre-image of $x$. To show that $p^{\prime}$ is injective suppose that $p\left(\left[\left(U_{n}^{1}\right)_{n}\right]\right)=p\left(\left[\left(U_{n}^{2}\right)_{n}\right]\right)$ so that $\left(V_{n}\right)_{n}$ defined by $V_{n}=U_{n}^{1} \cap U_{n}^{2}$ is in $\widetilde{\Gamma}$. Then $\left[\left(V_{n}\right)_{n}\right] \preceq\left[\left(U_{n}^{i}\right)_{n}\right]$ and by minimality $\left[\left(V_{n}\right)_{n}\right]=\left[\left(U_{n}^{i}\right)_{n}\right]$. It is clear that $p^{\prime-1}(U)=\mathcal{U}_{U}$ for $U \in \Gamma$. Thus $p^{\prime}$ is a homeomorphism. So it remains to show that $p^{\prime}$ preserves composability and $p^{\prime}\left(\left[\left(U_{n}\right)_{n}\right]\left[\left(V_{n}\right)_{n}\right]\right)=p^{\prime}\left(\left[\left(U_{n}\right)_{n}\right]\right) p^{\prime}\left(\left[\left(V_{n}\right)_{n}\right]\right)$, in case $\left[\left(U_{n}\right)_{n}\right] \vdash\left[\left(V_{n}\right)_{n}\right]$. Let $\left[\left(U_{n}\right)_{n}\right] \vdash\left[\left(V_{n}\right)_{n}\right]$. This is equivalent to $\left[\left(d(U)_{n}\right)_{n}\right]=\left[\left(r(U)_{n}\right)_{n}\right]$, and hence for $\{x\}=\bigcap_{n} U_{n}$ and $\{y\}=\bigcap_{n} V_{n}$ it implies $x \vdash y$. Moreover, in that case $x y \in \bigcap_{n} U_{n} V_{n}=p\left(\left[\left(U_{n}\right)_{n}\right]\left[\left(V_{n}\right)_{n}\right]\right)$, and since $p\left(\left[\left(U_{n}\right)_{n}\right]\left[\left(V_{n}\right)_{n}\right]\right)$ is a singleton the claim follows.

q.e.d.

A topological space which has a base consisting of closed (and open) sets is called zero dimensional. Hence the groupoid of the last theorem is zero dimensional. A zero dimensional $T_{1}$ space is totally disconnected, i.e. the only connected set containing a point $x$ is the singleton (one point set) containing $x$.

1.2.4. The universal groupoid of an inverse semigroup. The question of how to assign a groupoid $G(S)$ to an inverse semigroup $S$ in such a way that $S$ may be identified with a sub inverse semigroup of $\mathcal{A S G}(G(S))$ has been thoroughly addressed in [Pat93, Pat95]. In particular, a construction is presented which yields the universal groupoid $G_{u}(S)$ of an inverse semigroup $S$. We have not made use of Paterson's approach but followed different lines and therefore include a brief comparison for completion. This is best done by first presenting $\mathcal{R}(\Gamma)$ in the manner it has been presented in [Kel95b] for tiling almost-groupoids. 
There is a right action of $\Gamma$ on the space of units $\Omega=\mathcal{R}(\Gamma)^{0}$ by means of partial homeomorphisms. Let

$$
\Omega^{\vdash}:=\{(\widetilde{u}, c) \in \Omega \times \Gamma \mid r(c) \succeq \widetilde{u}\}
$$

with relative topology, $\Omega \times \Gamma$ carrying the product topology. Let $\gamma: \Omega^{\vdash} \rightarrow \Omega:(\widetilde{u}, c) \mapsto$ $d(\widetilde{u} c)$. Then $\gamma(\cdot, c): \mathcal{U}_{r(c)} \rightarrow \mathcal{U}_{d(c)}$ is a partial homeomorphism. Now consider the equivalence relation on $\Omega^{\vdash}$

$$
(\widetilde{u}, c) \sim\left(\widetilde{u}, c^{\prime}\right) \quad \text { whenever } \exists n: u_{n} c=u_{n} c^{\prime} .
$$

It is straightforward to see that this definition is independent of the choice of the representative $\left(u_{n}\right)_{n}$ of $\widetilde{u}$ and that the relation is transitive. We denote the equivalence class of $(\widetilde{u}, c)$ by $[\widetilde{u}, c]$.

Lemma 6. Let $\mathcal{R}^{\prime}(\Gamma)$ be quotient of $\Omega^{\vdash}$ by the above equivalence relation with quotient topology and consider the groupoid structure defined by $[\widetilde{u}, c]\left[\widetilde{u}^{\prime}, c^{\prime}\right]=\left[\widetilde{u}, c c^{\prime}\right]$ provided $\widetilde{u}^{\prime}=d(\widetilde{u} c)$ and $[\widetilde{u}, c]^{-1}=\left[d(\widetilde{u} c), c^{-1}\right]$. Then $\mathcal{R}^{\prime}(\Gamma)$ is a groupoid which is isomorphic to $\mathcal{R}(\Gamma)$.

Proof: Let $f: \Omega^{\vdash} \rightarrow \mathcal{R}(\Gamma), f(\widetilde{u}, c):=\widetilde{u} c . \quad f$ is surjective, since $\widetilde{c}=\widetilde{r(c)} c_{1}$ for some representative $\left(c_{n}\right)_{n}$ of $\widetilde{c}$. If $f(\widetilde{u}, c)=f\left(\widetilde{u}^{\prime}, c^{\prime}\right)$ then first, $\widetilde{u}=\widetilde{u}^{\prime}$, and second $\exists n: u_{n} \preceq r(c), r\left(c^{\prime}\right)$ so that $(\widetilde{u}, c)$ and $\left(\widetilde{u}, c^{\prime}\right)$ are equivalent in the above sense. The topology of $\mathcal{R}^{\prime}(\Gamma)$ is generated by sets of the form $\left[\mathcal{U}_{u} \times\{c\} \cap \Omega^{\vdash}\right]$. Such a set is equal to $\left[\mathcal{U}_{r(u c)} \times\{u c\}\right]=\{[\widetilde{u}, u c] \mid r(u c) \succeq \widetilde{u}\}$ in case $u \vdash c$ and otherwise empty. Since $f^{-1}\left(\mathcal{U}_{c}\right)=\mathcal{U}_{r(c)} \times\{c\}$ for any $c \in \Gamma, f$ induces a homeomorphism between $\mathcal{R}^{\prime}(\Gamma)$ and $\mathcal{R}(\Gamma)$. It is straightforward to check that this homeomorphism preserves multiplication and inversion.

q.e.d.

To compare this with the universal groupoid $G_{u}$ defined by $\Gamma_{0}$ [Pat93, Pat95] we assume that $\Gamma$ is countable. Paterson looks a the space $X$ of all nonzero semicharacters of $\Gamma_{0}^{0}$, i.e. at nonzero (inverse semigroup) homomorphisms $\alpha: \Gamma_{0}^{0} \rightarrow\{0,1\}$, the latter being a group under multiplication. Semicharacters yield an inverse semigroup under point-wise multiplication, but $X$ not containing the zero map, it is an almost-groupoid under point-wise multiplication. We denote by 1 the semicharacter which is identically to 1 .

Lemma 7. The map $\widetilde{\Gamma}^{0} \rightarrow X \backslash\{1\}: \widetilde{u} \mapsto \alpha_{\widetilde{u}}$ where $\alpha_{\widetilde{u}}(v)=1$ if and only if $v \succeq \widetilde{u}$ is an isomorphism of almost-groupoids (both containing only units).

Let $\widetilde{u} \vdash \widetilde{u}^{\prime}$ for two elements of $\widetilde{\Gamma}^{0}$. Then, for $v \in \Gamma_{0}^{0}, \widetilde{u} \widetilde{u}^{\prime} \preceq v$ is equivalent to $\widetilde{u} \preceq v$ and $\widetilde{u}^{\prime} \preceq v$. Hence $\alpha_{\widetilde{u} \widetilde{u}^{\prime}}=\alpha_{\widetilde{u}} \alpha_{\widetilde{u}^{\prime}}$. The above map is therefore a homomorphism which is obviously injective. Let $\alpha \in X, \alpha \neq 1$, and $\Gamma_{\alpha}^{0}=\left\{u \in \Gamma_{0}^{0} \mid \alpha(u)=1\right\}$ be the support of $\alpha . \quad \Gamma_{\alpha}^{0}$ is a sub-inverse semigroup of $\Gamma_{0}^{0}$ which is lower directed, i.e. any two of its elements have a lower bound in it. From the countability condition and Lemma 2 follows that $\widetilde{\Gamma^{0}}$ has a unique minimal element, call it $\widetilde{u}_{\alpha}$. Then $\alpha=\alpha_{\widetilde{u}_{\alpha}}$. $\quad$ q.e.d.

Identifying $X$ with $\widetilde{\Gamma}^{0} \cup\{1\}$, where we consider 1 as an extra element of $\widetilde{\Gamma}$ which satisfies $\forall \widetilde{c} \in \widetilde{\Gamma}: 1 \widetilde{c}=\widetilde{c}=\widetilde{c} 1$ and $11=1$, Paterson's topology can be described as the one which is generated by sets of the form

$$
A_{u ; u_{1}, \cdots, u_{k}}:=A_{u} \cap A_{u_{1}}^{c} \cap \cdots \cap A_{u_{k}}^{c}
$$


with $u, u_{i} \in \Gamma_{0}^{0}, u_{i} \preceq u, A_{u}=\widetilde{U}_{u} \cup\{1\}$, and $A_{u_{i}}^{c}$ here denoting the complement of $A_{u_{i}}$. In particular, the relative topology of this topology on $\widetilde{\Gamma}^{0}$ is finer then the one we consider.

The universal groupoid $G_{u}\left(\Gamma_{0}\right)$ is now obtained from a right action of $\Gamma$ on $X$. Define

$$
X^{\vdash}:=\left\{(\widetilde{u}, c) \in \widetilde{\Gamma}^{0} \times \Gamma \mid r(c) \succeq \widetilde{u}\right\} \cup\{1\} \times \Gamma
$$

with relative topology, $X \times \Gamma$ carrying the product topology. Let $\gamma: X^{\vdash} \rightarrow X$ with $\gamma(x, c)=d(x c)$. Again, $\gamma(\cdot, c): A_{r(c)} \rightarrow A_{d(c)}$ is a partial homeomorphism. Consider the equivalence relation on $X^{\vdash}$

$$
(\widetilde{u}, c) \sim\left(\widetilde{u}, c^{\prime}\right) \text { whenever } \exists n: u_{n} c=u_{n} c^{\prime},
$$

for $\widetilde{u} \in \widetilde{\Gamma}^{0}$, whereas $(1, c)$ is only equivalent to itself. Again, it is independent of the choice of representative and transitive. The universal groupoid $G_{u}\left(\Gamma_{0}\right)$ is given by the quotient of $X^{\vdash}$ w.r.t. the above equivalence relation with quotient topology and groupoid structure defined by $[x, c]\left[x^{\prime}, c^{\prime}\right]=\left[x, c c^{\prime}\right]$ provided $x^{\prime}=d(x c)$ and $[x, c]^{-1}=$ $\left[d(x c), c^{-1}\right]$, square brackets again denoting equivalence classes. We will have more to say about the relation between $\mathcal{R}(\Gamma)$ and $G_{u}\left(\Gamma_{0}\right)$ in the case where $\Gamma$ is a tiling almost-groupoid.

1.3. Application to tilings. Let us see what $\mathcal{R}$ yields applied to the almost-groupoid $\mathcal{M}_{\text {II }}$ of a tiling $T$. For that we consider a notion of radius of a doubly pointed pattern class. Let $\mathrm{rad}: \mathcal{M}_{\mathbb{I}} \rightarrow \mathbb{R}^{+}$be defined by the Euclidean distance between the two tiles of the ordered pair and the boundary of a pattern class 5 . In particular $\operatorname{rad}(c)=$ $\min \{\operatorname{rad}(r(c)), \operatorname{rad}(d(c))\}$ and $c \preceq c^{\prime}$ implies $\operatorname{rad}(c) \geq \operatorname{rad}\left(c^{\prime}\right)$. Furthermore, let $M_{r}(c)$, $r>0$, be the doubly pointed pattern class which is obtained from $c$ by eliminating all tiles which have distance greater than or equal to $r$ from both pointed tiles and $M_{0}(c)$ be the doubly pointed pattern class which is given by the pointed tiles only. The finite type (compactness) condition takes then the form

- The set $\left\{M_{r}(c) \mid c \in \mathcal{M}_{\mathbb{I}}\right\}$ is finite for any $r$.

Of particular interest are doubly pointed pattern classes called $r$-patches which are those which satisfy $c=M_{r}(c)$ and $\operatorname{rad}(c) \geq r$. Consider the metric on $\Gamma$ defined by

$$
\left.d\left(c, c^{\prime}\right)=\inf \left(\left\{e^{-r} \mid M_{r}(c)=M_{r}\left(c^{\prime}\right)\right\} \cup\left\{e^{-1}\right\}\right)\right) .
$$

Theorem 4. Let $\mathcal{M}_{\mathrm{II}}$ be the almost-groupoid of a tiling which satisfies the finite type condition. Then there is a continuous bijection between $\widetilde{\mathcal{M}}_{\mathbb{I I}}$ and the metric completion of $\mathcal{M}_{\mathrm{II}}$ with respect to the above metric. Furthermore, the sets $U_{c}, c \in \mathcal{M}_{\mathrm{II}}$ are metriccompact.

Proof: Let $\left(c_{n}\right)_{n}$ be a decreasing sequence of doubly pointed pattern classes. Since $c \succeq c^{\prime}$ implies $M_{r}(c) \succeq M_{r}\left(c^{\prime}\right)$ the finite type condition implies the existence of an $N$ such that for all $n \geq N: M_{r}\left(c_{n}\right)=M_{r}\left(c_{N}\right)$. It follows that $d\left(c_{n}, c_{m}\right) \leq e^{-r}$ for $n, m \geq N$, i.e. $\left(c_{n}\right)_{n}$ is a Cauchy sequence. Moreover, if $\left(c_{n}\right)_{n}$ and $\left(c_{n}^{\prime}\right)_{n}$ are two decreasing sequences which are equivalent in the sense (đ) a similar argument shows that $d\left(c_{n}, c_{n}^{\prime}\right) \rightarrow 0$, i.e. that they are equivalent as Cauchy sequences. Now fix an increasing sequence $\left(r_{k}\right)_{k}$ of positive numbers which diverges. If $\left(c_{n}\right)_{n}$ is a Cauchy

\footnotetext{
${ }^{5}$ Choosing a representative for the pattern class it is the Euclidean distance between the boundary of the subset it covers and the subset covered by the two tiles of the ordered pair.
} 
sequence then $\forall k \exists N_{k} \forall n \geq N_{k}: M_{r_{k}}\left(c_{N_{k}}\right)=M_{r_{k}}\left(c_{n}\right)$. Defining $j_{k}=j\left(\left(c_{n}\right)_{n}\right)_{k}=$ $M_{r_{k}}\left(c_{N_{k}}\right)$ yields thus a decreasing sequence for which $d\left(j_{k}, c_{N_{k}}\right) \rightarrow 0$, i.e. which is equivalent to $\left(c_{n}\right)_{n}$ as Cauchy sequence. Moreover, if $\left(c_{n}\right)_{n}$ and $\left(c_{n}^{\prime}\right)_{n}$ are Cauchy equivalent sequences then $j\left(\left(c_{n}\right)_{n}\right)=j\left(\left(c_{n}^{\prime}\right)_{n}\right)$. If $\left(c_{n}\right)_{n}$ is decreasing, then not only $j\left(\left(c_{n}\right)_{n}\right) \succeq\left(c_{n}\right)_{n}$, but since $\forall n \exists k: r_{k}>\operatorname{rad}\left(c_{n}\right)$ also $j\left(\left(c_{n}\right)_{n}\right) \preceq\left(c_{n}\right)_{n}$. So if $j\left(\left(c_{n}\right)_{n}\right)$ and $j\left(\left(c_{n}^{\prime}\right)_{n}\right)$ are not equivalent in the sense (4) they cannot belong to the same Cauchy class. Therefore is the map which sends $\widetilde{c}$ to its Cauchy class a well defined bijection between $\widetilde{\mathcal{M}_{\text {II }}}$ and the metric completion of $\mathcal{M}_{\mathbb{I}}$.

To compare the topologies extend $M_{r}$ to $\widetilde{\mathcal{M}_{\mathbb{I}}}$ through $M_{r}(\widetilde{c})=\lim _{n} M_{r}\left(c_{n}\right),\left[\left(c_{n}\right)_{n}\right]=$ $\widetilde{c}$. The limit exists and is independent of the chosen representative by the same argument as above which in fact shows that $\lim _{n} M_{r}\left(c_{n}\right)=M_{r}\left(c_{N}\right)$ for some $N$. It is then straightforward to check that the extension of the metric to the completion of $\mathcal{M}_{\mathbb{I I}}$ is given by formally the same expression for $d$ as in (14). Again using the finite type condition one sees that the image of the (continuous) function $d(\widetilde{c}, \cdot): \widetilde{\mathcal{M}_{I}} \rightarrow \mathbb{R}^{+}$ is discrete apart from a limit point at 0 . Therefore $\epsilon$-neighbourhoods are closed and hence complete in the metric topology. $\epsilon$-neighbourhoods are sets of the form

$$
U_{r}(\widetilde{c})=\left\{\widetilde{c} \mid M_{r}(\widetilde{c})=M_{r}(\widetilde{c})\right\}
$$

(the smaller $\epsilon$ the bigger $r$ ) but since $r$ is finite $U_{r}(\widetilde{c})=U_{r}\left(c_{n}\right)$ for some $n$ and representative $\left(c_{n}\right)_{n}$. If $0<r_{1}<r_{2}$ then $U_{r_{1}}(c)=\bigcup_{c^{\prime} \mid M_{r_{1}}\left(c^{\prime}\right)=M_{r_{1}}(c)} U_{r_{2}}\left(c^{\prime}\right)$ but by the finite type condition only finitely many sets in the union of the r.h.s. are mutually disjoint. Thus for any $0<\epsilon_{2}<\epsilon_{1}$ holds that the $\epsilon_{1}$-neighbourhood has a finite cover by $\epsilon_{2}$-neighbourhoods, i.e. $\epsilon$-neighbourhoods are pre-compact and hence compact.

If $c$ is an $r$-patch then $U_{r}(c)=U_{c}$. For arbitrary $c \in \mathcal{M}_{\mathbb{I I}}$ one has $U_{c}=\bigcup_{c^{\prime}}{ }_{c} U_{r}\left(c^{\prime}\right)$ where $r$ is some number bigger than the diameter of $c$ (the diameter of the set covered by a representative of the pattern class in $\mathbb{R}^{d}$ ). In particular, the metric topology is finer than the original topology on $\widetilde{\mathcal{M}_{\mathbb{I I}}}$. But moreover, only finitely many sets in the union of the r.h.s. are mutually disjoint so that the $U_{c}$ are metric-compact. q.e.d.

To proceed let us extend the radius function $\operatorname{rad}: \widetilde{\mathcal{M}_{\mathbb{I}}} \rightarrow \mathbb{R}^{+} \cup\{\infty\}$ through $\operatorname{rad}(\widetilde{x})=$ $\lim _{n} \operatorname{rad}\left(x_{n}\right)$ the r.h.s. being independent of the representative.

Lemma 8. Let $\mathcal{M}_{\mathbb{I}}$ be the almost-groupoid of a tiling which satisfies the finite type condition. $\widetilde{c}$ is minimal if and only if $\operatorname{rad}(\widetilde{c})=\infty$. Stated differently, a sequence $\left(c_{n}\right)_{n} \in \mathcal{M}_{\mathbb{I}} \underset{\mathbb{N}}{\mathbb{N}}$ is approximating if and only if the sequence $\left(\operatorname{rad}\left(c_{n}\right)\right)_{n}$ diverges.

Proof: Suppose that $\operatorname{rad}(\widetilde{c})=R^{\prime}<\infty$ and let $R>R^{\prime}$. There is at least one but at most finitely many $R$-patches $d_{1}, \ldots, d_{k}$ for which $d_{i} \preceq M_{R}(\widetilde{c})$. Now consider the sequence which is obtained from a representative $\left(c_{n}\right)_{n}$ of $\widetilde{c}$ by replacing each $c_{n}$ by $k$ elements $r\left(d_{1}\right) c_{n}, \ldots, r\left(d_{k}\right) c_{n}$. Since $U_{c_{1}}$ is metric-compact the sequence has a metric-convergent subsequence, say $\left(c_{n}^{\prime}\right)_{n}$, which we may assume to be decreasing (if not apply the map $j$ defined in the proof of Theorem 4). But then $\left(c_{n}^{\prime}\right)_{n} \preceq\left(c_{n}\right)_{n}$ and since $\operatorname{rad}\left(\left(c_{n}^{\prime}\right)_{n}\right) \geq R,\left(c_{n}^{\prime}\right)_{n}$ cannot be equivalent to $\left(c_{n}\right)_{n}$. Hence $\widetilde{c}$ is not minimal.

For the converse suppose that $\operatorname{rad}\left(c_{n}\right)$ diverges and $\left(c_{n}^{\prime}\right)_{n} \preceq\left(c_{n}\right)_{n}$. Since for all $n$ there is an $m$ such that $\operatorname{rad}\left(c_{m}\right)$ is larger than the diameter of $c_{n}^{\prime}$ this implies $c_{n}^{\prime} \succeq c_{m}$ and thus $\left(c_{n}^{\prime}\right)_{n} \succeq\left(c_{n}\right)_{n}$. Note that we do not need the finite type condition for this part.

q.e.d. 
Lemma 9. Let $\mathcal{M}_{\mathbb{I I}}$ be the almost-groupoid of a tiling which satisfies the finite type condition. Then the relative topologies on $\mathcal{R}\left(\mathcal{M}_{\mathbb{I}}\right)$ coincide and $\mathcal{R}\left(\mathcal{M}_{\mathbb{I I}}\right)$ is metricclosed in $\widetilde{\mathcal{M}_{\text {II }}}$.

Proof: The relative metric-topology on $\mathcal{R}\left(\mathcal{M}_{\mathbb{I I}}\right)$ is generated by sets $U_{r}(\widetilde{c}) \cap \mathcal{R}\left(\mathcal{M}_{\mathbb{I}}\right)$ where $\operatorname{rad}(\widetilde{c})=\infty$. Hence $M_{r}(\widetilde{c})=M_{r}\left(c^{\prime}\right)$ for some $r$-patch $c^{\prime}$ and thus $U_{r}(\widetilde{c})=U_{c^{\prime}}$. This shows that $U_{r}(\widetilde{c}) \cap \mathcal{R}\left(\mathcal{M}_{\mathbb{I}}\right)$ is open with respect to the original topology on $\mathcal{R}\left(\mathcal{M}_{\mathbb{I}}\right)$, i.e. the latter is finer than the relative metric-topology. By Theorem the topologies coincide.

Now suppose that $\widetilde{x}$ is not minimal, i.e. $\operatorname{rad}(\widetilde{x})=R^{\prime}<\infty$. Let $R>R^{\prime}$ and $\widetilde{y}$ be an element of the $e^{-R}$-neighbourhood of $\widetilde{x}$. Then $\operatorname{rad}(\widetilde{y})=R^{\prime}$ as well, and hence $\widetilde{y}$ is not minimal, i.e. $\widetilde{\mathcal{M}_{\text {II }}} \backslash \mathcal{R}\left(\mathcal{M}_{\mathbb{I I}}\right)$ is metric open.

q.e.d.

Corollary 1. Under the requirements of Theorem $\mathbb{6}$ is $\mathcal{U}_{c}$ compact. In particular is $\mathcal{R}\left(\mathcal{M}_{\mathbb{I I}}\right)^{0}$ a compact zero dimensional metric space and $\beta_{o}\left(\mathcal{R}\left(\mathcal{M}_{\mathbb{I I}}\right)\right)$ a sub-inverse semigroup of $\mathcal{A S G}\left(\mathcal{R}\left(\mathcal{M}_{\mathbb{I I}}\right)\right)$.

The compactness of $\mathcal{U}_{c}$ follows from Theorem 1 and Lemma 9. Writing $\mathcal{R}\left(\mathcal{M}_{\mathrm{II}}\right)^{0}=$ $\bigcup_{u} \mathcal{U}_{u}$, the union being taken over all $u \in \mathcal{M}_{\mathrm{I}}$ which consists only of one tile shows that the finite type condition implies compactness for $\mathcal{R}\left(\mathcal{M}_{\mathbb{I}}\right)^{0}$.

Roughly speaking, we have shown that the elements of $\mathcal{R}\left(\mathcal{M}_{\mathbb{I}}\right)$ can be seen as limits of doubly pointed pattern classes whose radii eventually become infinite. This can be formulated as follows: To a given approximating sequence $\left(c_{n}\right)_{n}$ construct a covering of $\mathbb{R}^{d}$ by first choosing a representative $\hat{c}_{1}$ for $c_{1}$ in $\mathbb{R}^{d}$. Then there are unique representatives $\hat{c}_{n}$ for $c_{n}$ such that $\hat{c}_{n}$ is obtained from $\hat{c}_{1}$ by addition of tiles (but keeping the ordered pair fixed). Since $\operatorname{rad}\left(c_{n}\right)$ diverges $\bigcup_{n} \hat{c}_{n}$ is a covering of $\mathbb{R}^{d}$ (each $\hat{c}_{n}$ is a set of tiles) together with an ordered pair of tiles. We call this a doubly pointed tiling. The elements of $\mathcal{R}\left(\mathcal{M}_{\mathbb{I}}\right)$ are the classes of doubly pointed tilings which are obtained in this way. The set of units $\Omega=\mathcal{R}\left(\mathcal{M}_{\mathbb{I}}\right)^{0}=\mathcal{R}\left(\mathcal{M}_{\mathrm{I}}\right)$ can than be identified with classes of tilings together with one chosen tile. It is called the hull of the tiling.

The relative Paterson topology on $\Omega$, c.f. (11), coincides with the topology on $\Omega$ considered above, since the sets $\mathcal{U}_{u}, u \in \mathcal{M}_{\mathrm{II}}^{0}$, which generate the latter are closed. Moreover, since

$$
\widetilde{U}_{r}(c)=\widetilde{U}_{M_{r}(c)} \backslash \bigcup_{r \text {-patches } c^{\prime} \neq c, c^{\prime} \preceq c} \widetilde{U}_{M_{r}\left(c^{\prime}\right)}
$$

the relative Paterson topology on $\widetilde{\Gamma}^{0}$ is finer than the metric topology and hence $\Omega$ is a Paterson-closed subset of $X$. It follows that $\mathcal{R}^{\prime}\left(\mathcal{M}_{\mathbb{I}}\right)$ is a reduction of the universal groupoid $G_{u}\left(\mathcal{M}_{\mathrm{II}} \cup\{0\}\right)$ with respect to the subset $\Omega$, which fits well into the general theory of [Pat95].

For later use we proof:

Lemma 10. Let $\mathcal{M}_{\mathbb{I}}$ be the almost-groupoid of a tiling which satisfies the finite type condition. Then any $\widetilde{c} \in \widetilde{\Gamma}$ has a smaller minimal element.

Proof: Suppose that $\widetilde{c}$ is not minimal and therefore $\operatorname{rad}(\widetilde{c})=R<\infty$. Fix an increasing diverging sequence of real numbers $\left(r_{k}\right)_{k}$ which are greater than $R$. As in the proof of Lemma 8 we construct $\widetilde{c}_{k}^{\prime}$ such that $\widetilde{c}_{k} \preceq \widetilde{c}$ and $\operatorname{rad}\left(\widetilde{c}_{k}\right) \geq r_{k}$. Hence 
$\widetilde{c}_{k} \in U_{r}(\widetilde{c})$ and since the latter is metric-compact the sequence $\left(\widetilde{c}_{k}^{\prime}\right)_{k}$ has a metricconvergent subsequence converging to a class $\widetilde{c}$ which is smaller than $\widetilde{c}$ and minimal. q.e.d.

1.3.1. A continuous groupoid associated to the tiling. There is another topological groupoid one can assign to a tiling, which we want to mention for comparison. Here one starts with the local isomorphism class $\mathcal{L}_{\mathcal{T}}$ of a tiling $\mathcal{T}$. This is the space of all tilings which are locally isomorphic to $\mathcal{T} . \mathcal{L}_{\mathcal{T}}$ may be obtained as the closure of the orbit of $\mathcal{T}$ under the action of the group $\mathbb{R}^{d}$ of translations with respect to a metric. In fact, viewed as a geometrical object a tiling may be translated, $\mathcal{T}-x, x \in \mathbb{R}^{d}$ is the covering given by the sets $t-x:=\{y-x \mid y \in t\}$ where $t$ runs through all tiles of $\mathcal{T}$. Then $\mathcal{L}_{\mathcal{T}}$ is the closure of $\left\{\mathcal{T}-x \mid x \in \mathbb{R}^{d}\right\}$ under the metric

$$
d\left(T, T^{\prime}\right)=\inf \left(\left\{\left\{\epsilon\left|\exists x, x^{\prime} \in \mathbb{R}^{d}: r\left(T-x, T^{\prime}-x^{\prime}\right) \geq \frac{1}{\epsilon},\right| x|,| x^{\prime} \mid<\epsilon\right\} \cup\left\{\frac{1}{\sqrt{2}}\right\}\right)\right.
$$

where $r\left(T, T^{\prime}\right)$ is the largest $r$ such that $T$ and $T^{\prime}$ agree on the $r$-ball around 0 [AP95. The other groupoid which may now be assigned to $\mathcal{T}$ is the transformation group $\mathcal{C}_{\mathcal{T}}:=\mathcal{L}_{\mathcal{T}} \times \mathbb{R}^{d}$. Two of its elements $(T, x),\left(T^{\prime}, x^{\prime}\right)$ are composable whenever $T^{\prime}=T-x$ and then $(T, x)\left(T^{\prime}, x^{\prime}\right)=\left(T, x+x^{\prime}\right)$. The topology is the product topology. For distinction we call it the continuous groupoid assigned to the tiling as opposed to the discrete one. How is it related to $\mathcal{R}\left(\mathcal{M}_{\mathbb{I}}(\mathcal{T})\right)$ ?

Fix for each tile-class a point in its interior, we call it a puncture. The punctures of the tiles of a tiling may be identified with a countable subset of $\mathbb{R}^{d}$. Let $\Omega_{\mathcal{T}}$ be the subset of $\mathcal{L}_{\mathcal{T}}$ which consists of tilings with the property that the puncture of one of its tiles identifies with $0 \in \mathbb{R}^{d}$. The reduction of $\mathcal{C}_{\mathcal{T}}$ by $\Omega_{\mathcal{T}}$, which is the sub-groupoid $\left\{(T, x) \in \mathcal{C}_{\mathcal{T}} \mid T, T-x \in \Omega\right\}$, is the groupoid which has been associated to an aperiodic tiling in $\mathrm{Kel950}$ It is isomorphic to $\mathcal{R}\left(\mathcal{M}_{\mathbb{I}}(\mathcal{T})\right)$, an isomorphism is given by the map which assigns to $(T, x)$ the doubly pointed tiling class which is given by the class of $T$ and the pair of tiles given by, first, the one which covers 0 , and second, the one which covers $x$.

Moreover, it has been proven by Anderson and Putnam AP95 that the above reduction of $\mathcal{C}_{\mathcal{T}}$ is an abstract transversal of $\mathcal{C}_{\mathcal{T}}$ in the sense of Muhly et al. so that by the work of the latter authors MRW87 the groupoid- $C^{*}$-algebras of $\mathcal{C}_{\mathcal{T}}$ and $\mathcal{R}\left(\mathcal{M}_{\mathbb{I I}}(\mathcal{T})\right)$ are stably isomorphic.

\section{Topological EQUiVAlEnCE AND MUtual LOCAL DERIVABILITY}

If we focus on the role tilings play in solid state physics when describing spatial structures, then several properties of the tiling are unimportant. First of all, only the congruence class of the tiling matters, and second, due to the locality of the interactions locally isomorphic tilings are equally well suited to describe that structure. This can now all be taken into account by working with the almost-groupoid of the tiling. However, investigating further the way how tilings model e.g. the arrangement of atoms (or ions) in solids one may take the point of view that this should only be understood in a topological way. In particular details like the precise position and strength of the bondings are to be added, i.e. are not to be derived from the tiling. This led Baake et al. from the theoretical physics group in Tübingen to introduce another equivalence 
relation between tilings which is based on mutual local derivability [BSJ91], see also BS95 for an overview.

Let $B_{r}(x)$ denote the closed $r$-ball around $x$ and $B_{r}=B_{r}(0)$. Furthermore $\mathcal{T} \sqcap B_{r}(x)$ is the pattern consisting of all tiles of $\mathcal{T}$ which intersect with $B_{r}(x)$.

Definition 6. $\mathcal{T}_{2}$ is locally derivable from $\mathcal{T}_{1}$ if there is an $r \geq 0$ such that for all $x, y \in \mathbb{R}^{d}$

$$
\left(\mathcal{T}_{1}-x\right) \sqcap B_{r}=\left(\mathcal{T}_{1}-y\right) \sqcap B_{r} \quad \text { implies } \quad\left(\mathcal{T}_{2}-x\right) \sqcap\{0\}=\left(\mathcal{T}_{2}-y\right) \sqcap\{0\} .
$$

Restricting our interest to tilings which satisfy the finite type condition the knowledge of the correspondence between $\left(\mathcal{T}_{1}-x\right) \sqcap B_{r}$ and $\left(\mathcal{T}_{2}-x\right) \sqcap\{0\}$ for finitely many $x$ is enough to construct all tiles of $\mathcal{T}_{2}$ from $\mathcal{T}_{1}$. This obviously defines a map $\ell: \mathcal{L}\left(\mathcal{T}_{1}\right) \rightarrow \mathcal{L}\left(\mathcal{T}_{2}\right)$, which is continuous, has dense image and is therefore surjective. $\ell$ can be extended to a surjective homomorphism of groupoids, $\ell: \mathcal{C}_{\mathcal{T}} \rightarrow \mathcal{C}_{\mathcal{T}^{\prime}}$ : $(T, x) \mapsto(\ell(T), x)$. We may call the replacement of $\mathcal{T}_{1} \sqcap B_{r}(x)$ by $\mathcal{T}_{2} \sqcap\{x\}$ a local derivation rule. In particular the above definition is equivalent to saying that for all $r^{\prime} \geq 0$ there is an $r \geq 0$ such that for all $x, y \in \mathbb{R}^{d}$

$$
\left(\mathcal{T}_{1}-x\right) \sqcap B_{r}=\left(\mathcal{T}_{1}-y\right) \sqcap B_{r} \quad \text { implies } \quad\left(\mathcal{T}_{2}-x\right) \sqcap B_{r^{\prime}}=\left(\mathcal{T}_{2}-y\right) \sqcap B_{r^{\prime}} .
$$

$\mathcal{T}_{1}$ and $\mathcal{T}_{2}$ are called mutually locally derivable if $\mathcal{T}_{2}$ is locally derivable from $\mathcal{T}_{1}$ and vice versa. This is an equivalence relation which can be extended by saying that $\mathcal{T}_{1}$ and $\mathcal{T}_{2}$ belong to the same MLD-class if there is a $\mathcal{T}_{2}^{\prime}$ which is locally isomorphic to $\mathcal{T}_{2}$ and mutually locally derivable from $\mathcal{T}_{1}$. That this extension is an equivalence relation (in fact on LI-classes) follows from the observation that if $\mathcal{T}_{2}$ is locally derivable from $\mathcal{T}_{1}$ and $\mathcal{T}_{1}^{\prime}$ is locally isomorphic to $\mathcal{T}_{1}$ then the local derivation rule can be used to locally derive a tiling $\mathcal{T}_{2}^{\prime}$ from $\mathcal{T}_{1}^{\prime}$. Then $\mathcal{T}_{2}^{\prime}$ has to be locally isomorphic to $\mathcal{T}_{2}$. Moreover, the local derivation of $\mathcal{T}_{1}$ from $\mathcal{T}_{2}^{\prime}$ yields the inverse of $\ell: \mathcal{C}_{\mathcal{T}} \rightarrow \mathcal{C}_{\mathcal{T}}$, so that the latter becomes an isomorphism.

Corollary 2. If $\mathcal{T}$ and $\mathcal{T}^{\prime}$ are in the same $M L D$-class then the groupoids $\mathcal{R}\left(\mathcal{M}_{\mathbb{I}}(\mathcal{T})\right.$ ) and $\mathcal{R}\left(\mathcal{M}_{\mathbb{I}}\left(\mathcal{T}^{\prime}\right)\right)$ are reductions (in fact abstract transversals) of the same groupoid. In particular they have stably isomorphic groupoid-C* $C^{*}$-algebras.

This follows directly from the fact that $\mathcal{C}_{\mathcal{T}}$ and $\mathcal{C}_{\mathcal{T}^{\prime}}$ are isomorphic and the above mentioned theorem of [AP95].

The above corollary indicates that the Tübingen formulation of local derivability is a good starting point to answer the question under which circumstances $\mathcal{R}\left(\mathcal{M}_{\mathbb{I I}}(\mathcal{T})\right)$ and $\mathcal{R}\left(\mathcal{M}_{\mathbb{I}}\left(\mathcal{T}^{\prime}\right)\right)$ are isomorphic. In order to cast it in a form applicable to our framework, using almost-groupoids and the discrete groupoid, we are naturally led to strengthen and at the same time to generalize the concept of local derivation. A strengthening comes along with the idea of preservation of the average number of tiles per unit volume whereas a generalization is necessary as we want to work in a purely topological setting.

2.1. Constructing local morphisms from local derivation rules. Suppose that $\mathcal{N}$ is a sub-almost-groupoid of $\Gamma$ which is the order ideal generated by a finitely generated almost-groupoid, i.e. $\mathcal{N}=I(\langle\mathcal{C}\rangle)$ where $\mathcal{C}$ is a finite set and $\langle\mathcal{C}\rangle$ the almostgroupoid generated by it. Suppose furthermore that we have a map $\hat{\varphi}: \mathcal{C} \rightarrow \Gamma^{\prime}$ which satisfies conditions which arise if it were the restriction of a prehomomorphism from 
$\mathcal{N}$ into an almost-groupoid $\Gamma^{\prime}$. A question which is of prime importance for sequel is whether we can construct a local morphism $\varphi: \mathcal{N} \rightarrow \Gamma^{\prime}$ from that map.

We call $n$ elements $c_{1}, \ldots, c_{n}$ collatable if they may be composed, i.e. if $\forall 1 \leq k<n$ : $c_{1} \ldots c_{k} \vdash c_{k+1}$. Let $\mathcal{C}^{-1}=\mathcal{C}$ be a finite subset of an almost-groupoid and $\hat{\varphi}: \mathcal{C} \rightarrow \Gamma^{\prime}$ be a map into another almost-groupoid which satisfies for all $c, c_{i} \in \mathcal{C}$ :

E1 $\hat{\varphi}\left(c^{-1}\right)=\hat{\varphi}(c)^{-1}$,

E2 if $c_{1}, \ldots, c_{n}$ are collatable then $\hat{\varphi}\left(c_{1}\right), \ldots, \hat{\varphi}\left(c_{n}\right)$ are collatable,

E3 if $c_{1} \ldots c_{n}$ is a unit then $\hat{\varphi}\left(c_{1}\right) \ldots \hat{\varphi}\left(c_{n}\right)$ is a unit.

Consider for $c \in\langle\mathcal{C}\rangle$

$$
\Phi(c)=\left\{\hat{\varphi}\left(c_{1}\right) \ldots \hat{\varphi}\left(c_{n}\right) \mid c_{1} \ldots c_{n}=c, c_{i} \in \mathcal{C}\right\} .
$$

Since $c_{1} \ldots c_{n}=c_{1}^{\prime} \ldots c_{n^{\prime}}^{\prime}$ implies that $\hat{\varphi}\left(c_{1}\right) \ldots \hat{\varphi}\left(c_{n}\right)\left(\hat{\varphi}\left(c_{1}^{\prime}\right) \ldots \hat{\varphi}\left(c_{n^{\prime}}^{\prime}\right)\right)^{-1}$ is a unit any two elements of $\Phi(c)$ have a common smaller element, i.e. $\Phi(c)$ is a lower directed set. Provided $\Phi(c)$ is finite we define

$$
\varphi(c):=\min \Phi(c) .
$$

Then $\varphi$ commutes with the inverse map, because of $\Phi(c)^{-1}=\Phi\left(c^{-1}\right)$, and it satisfies inequality (6) since $\Phi\left(c_{1}\right) \Phi\left(c_{2}\right) \subset \Phi\left(c_{1} c_{2}\right)$. Thus $\varphi:\langle\mathcal{C}\rangle \rightarrow \Gamma^{\prime}$ is a prehomomorphism. If $H_{\mathcal{C}}(c):=\left\{c^{\prime} \in\langle\mathcal{C}\rangle \mid c^{\prime} \succeq c\right\}$ has a unique minimal element then $\pi: I(\langle\mathcal{C}\rangle) \rightarrow\langle\mathcal{C}\rangle$ : $\pi(c)=\min H_{\mathcal{C}}(c)$ is a prehomomorphism as well, and we may extend $\varphi$ through $\varphi \circ \pi$.

Definition 7. We call a pair $(\varphi, \mathcal{C})$, where $\mathcal{C}=\mathcal{C}^{-1} \subset \Gamma$ is finite and $\hat{\varphi}: \mathcal{C} \rightarrow \Gamma^{\prime}$ satisfies conditions E1-3, a local derivation rule if it leads for all $c \in \Gamma$ to finite lower directed sets $\Phi(c)$ and $H_{\mathcal{C}}(c)$ and $\varphi: I(\langle\mathcal{C}\rangle) \rightarrow \Gamma^{\prime}$,

$$
\varphi(c):=\min \Phi\left(\min H_{\mathcal{C}}(c)\right)
$$

is approximating.

Lemma 11. Let $\mathcal{M}_{\mathbb{I}}$ and $\mathcal{M}_{\mathbb{I I}}^{\prime}$ be two tiling almost-groupoids. Suppose that there exist a finite $\mathcal{C}=\mathcal{C}^{-1} \subset \mathcal{M}_{\mathbb{I I}}$ and a map $\hat{\varphi}: \mathcal{C} \rightarrow \mathcal{M}_{\mathrm{II}}^{\prime}$ which satisfies E1-3. Then $\Phi(c)$ and $H_{\mathcal{C}}(c)$ are finite lower directed sets.

Proof: $H_{\mathcal{C}}(c)$ is finite since any doubly pointed pattern class has only finitely many tiles. It is lower directed since $\mathcal{M}_{\mathbb{I}}$ is unit hereditary. As for $\Phi(c)$ we subdivide this set first into subsets $c^{\prime} \Phi_{c^{\prime} c^{\prime \prime}}(c) c^{\prime \prime}$ where $\Phi_{c^{\prime} c^{\prime \prime}}(c):=\left\{\hat{\varphi}\left(u_{1}\right) \ldots \hat{\varphi}\left(u_{n}\right) \mid n \in \mathbb{N}, c=\right.$ $\left.c^{\prime} u_{1} \ldots u_{n} c^{\prime \prime}\right\}, u_{i} \in\langle\mathcal{C}\rangle^{0}$ and $c^{\prime}=c_{1}^{\prime} \cdots c_{k}^{\prime}, c_{i}^{\prime} \in \mathcal{C}$ none of the $c_{i}^{\prime} \cdots c_{j}^{\prime}, 1 \leq i \leq j \leq k$, being a unit, and the same conditions for $c^{\prime \prime}$. Since there are only finitely many different units which satisfy $u \succeq c^{\prime-1} c c^{\prime \prime-1}$, units commute, and $\varphi(u) \varphi(u)=\varphi(u), \Phi_{c^{\prime} c^{\prime \prime}}(c)$ is finite. Moreover, there are only finitely many different possibilities to choose $c^{\prime}, c^{\prime \prime}$ so that $\Phi(c)$ is finite.

q.e.d.

There is no reason why $\varphi$ should be approximating.

To connect the Tübingen formulation of local derivability with the above and justify double use of the word local derivation rule we proof:

Theorem 5. Let $\mathcal{T}^{\prime}$ be locally derivable from $\mathcal{T}$. Then there exists a local derivation rule in the sense of Definition $\bar{\gamma}, \hat{\varphi}: \mathcal{C} \subset \mathcal{M}_{\mathbb{I}}(\mathcal{T}) \rightarrow \mathcal{M}_{\mathbb{I}}\left(\mathcal{T}^{\prime}\right)$, such that $\mathcal{R}(I(\langle\mathcal{C}\rangle))=$ $\mathcal{R}\left(\mathcal{M}_{\mathbb{I}}(\mathcal{T})\right)$ and the induced homomorphism maps the class of $\mathcal{T}$ onto that of $\mathcal{T}^{\prime}$. 
Proof: First introduce punctures for the tile classes of $\mathcal{T}$ which are chosen such that none of the punctures of tiles of $\mathcal{T}$ lies on the boundary of tiles of $\mathcal{T}^{\prime}$. For given $r^{\prime}$ fix $r$ according to (16) and let for any tile $t$ of $\mathcal{T}, \hat{\ell}(t)=\mathcal{T}^{\prime} \sqcap B_{r^{\prime}}\left(t^{p c t}\right)$, where $t^{p c t}$ is the puncture of $t$. We now define a local derivation rule on the set $\mathcal{C}_{r}^{c}$ of all $r$-patches $c$ for which $M_{0}(c)$ is connected. Let $m$ be a doubly pointed pattern in $\mathcal{T}$ of the class $\widetilde{m} \in \mathcal{C}_{r}^{c}$. Denote the $i$ th tile of its ordered pair by $t_{i}(m)$. Then $\hat{\varphi}(\widetilde{m})$ shall be the class of the pattern $\hat{\ell}\left(t_{1}(m)\right) \cup \hat{\ell}\left(t_{2}(m)\right)$ with the ordered pair $\left(\mathcal{T}^{\prime} \sqcap B_{0}\left(t_{1}(m)^{p c t}\right), \mathcal{T}^{\prime} \sqcap B_{0}\left(t_{2}(m)^{p c t}\right)\right)$. That $\hat{\varphi}(\widetilde{m})$ does not depend on the chosen representative $m$ for $\widetilde{m}$ is precisely the definition of local derivability. Defined in that geometrical way, it is easy to see that $\hat{\varphi}$ satisfies the conditions E1-3. If $r^{\prime}$ is larger than twice the diameter of the largest tile in $\mathcal{T}_{1}$ then $\hat{\ell}\left(t_{1}(m)\right) \cup \hat{\ell}\left(t_{2}(m)\right)$ is connected and $\varphi$ approximating. By construction it maps the class of $\mathcal{T}$ onto that of $\mathcal{T}^{\prime}$.

q.e.d.

Although the local derivation rule $\hat{\varphi}$ yields a homomorphism $\mathcal{R}(\varphi)$ which is very similar to a restriction of the map $\ell: \mathcal{C}_{\mathcal{T}} \rightarrow \mathcal{C}_{\mathcal{T}}$, constructed from the local derivation rule in the Tübingen version it is neither injective nor surjective, in general. The geometrical picture of $\ell: \mathcal{C}_{\mathcal{T}} \rightarrow \mathcal{C}_{\mathcal{T}}$, allows one to conclude that $\mathcal{R}(\varphi)$ is surjective whenever the punctures for the tiles of $\mathcal{T}$ can be chosen in such a way that any tile of $\mathcal{T}^{\prime}$ contains at least one puncture. (First, doubly pointed tiling classes $\widetilde{c} \in \mathcal{R}\left(\mathcal{M}_{\mathbb{I}}\left(\mathcal{T}^{\prime}\right)\right.$ ) for which $r(\widetilde{c})$ is in the same class then $\mathcal{T}^{\prime}$ lie in the image of $\mathcal{R}(\varphi)$, and then, by continuity, all of $\mathcal{R}\left(\mathcal{M}_{\mathbb{I I}}\left(\mathcal{T}^{\prime}\right)\right)$.) Similarly, a necessary (but not sufficient) condition for $\mathcal{R}(\varphi)$ to be injective is that any tile of $\mathcal{T}^{\prime}$ contains at most one puncture. Hence the failure of $\mathcal{R}(\varphi)$ to be an isomorphism may have its cause in that the average number of tiles per unit volume is not preserved.

The converse of the theorem is false. If $\mathcal{T}^{\prime}$ is obtained from $\mathcal{T}$ by a change of length scale or an overall rotation there would (apart from symmetric cases) not be a local derivation rule in the Tübingen sense but a local derivation in the sense of Definition 7 is is given by applying the change of length scale resp. rotation to the doubly pointed pattern classes.

2.2. Topological equivalence. An answer to the question under which circumstances two tilings lead to isomorphic groupoids shall be given here in purely "local" terms, i.e. in terms of almost-groupoids and local derivation rules. For that let us start with a lemma. Let us use the notation that for subsets of an ordered set $X \preceq Y$ if $\forall y \in Y \exists x \in X: x \preceq y$.

Lemma 12. Let $\varphi$ be a local morphism from a countable unit hereditary almost-groupoid $\Gamma$ into itself. Then $\mathcal{R}(\varphi)=$ id if an only if $D(\varphi) \preceq \Gamma$ and $\varphi(c)$ and $c$ have for all $c \in D(\varphi)$ a lower bound.

Proof: Suppose first that $\mathcal{R}(\varphi)=$ id which in particular means $\mathcal{R}(D(\varphi))=\mathcal{R}(\Gamma)$. Let $c \in \Gamma$, by Lemma 2 there is a smaller minimal element $\left[\left(c_{n}\right)_{n}\right]$. It has a representative $\left(c_{n}\right)_{n}, c_{n} \in D(\varphi)$. But then there exists already some $c_{n} \in D(\varphi)$ for which $c_{n} \preceq c$. Furthermore, $\varphi\left(c_{n}\right)$ and $c_{n}$ must have for any $n$ a lower bound since they constitute equivalent sequences. Any such bound is also a lower bound for $\varphi(c)$ and $c$.

As for the converse observe that under the assumption that $\varphi(c)$ and $c$ have a lower bound for all $c \in D(\varphi)$ we have $\widetilde{\varphi}(\widetilde{c}) d(\widetilde{c}) \preceq \widetilde{\varphi}(\widetilde{c}), \widetilde{c}$ and hence for minimal $\widetilde{c}$ : $\widetilde{\varphi}(\widetilde{c})=$ $\widetilde{c}$. Hence $\mathcal{R}(\varphi)=\left.\mathrm{id}\right|_{\mathcal{R}(D(\varphi))}$. But since $D(\varphi)$ is an order ideal, $D(\varphi) \preceq \Gamma$ implies $\mathcal{R}(D(\varphi))=\mathcal{R}(\Gamma)$ 
Definition 8. Two countable unit hereditary almost-groupoids $\Gamma$ and $\Gamma^{\prime}$ are called topologically equivalent if there are local derivation rules $\hat{\varphi}: \mathcal{C} \subset \Gamma \rightarrow \Gamma^{\prime}, \hat{\psi}: \mathcal{C}^{\prime} \subset \Gamma^{\prime} \rightarrow \Gamma$ such that for the induced local morphisms $\varphi$ resp. $\psi$ holds $D(\psi \circ \varphi) \preceq \Gamma, D(\varphi \circ \psi) \preceq \Gamma^{\prime}$, and $\psi(\varphi(c))$ and $c$ have for all $c \in D(\psi \circ \varphi)$ resp. $\varphi\left(\psi\left(c^{\prime}\right)\right)$ and $c^{\prime}$ for all $c^{\prime} \in D(\varphi \circ \psi)$ a lower bound. Two tilings of finite type are called topologically equivalent if their corresponding almost-groupoids are topologically equivalent.

According to the above lemma the definition of topological equivalence may equally well be formulated by saying that the local morphisms $\varphi$ and $\psi$ satisfy $\mathcal{R}(\psi \circ \varphi)=$ id on $\mathcal{R}(\Gamma)$ and $\mathcal{R}(\varphi \circ \psi)=$ id on $\mathcal{R}\left(\Gamma^{\prime}\right)$. By the functorial properties of $\mathcal{R}$ it implies that $\mathcal{R}(\Gamma)$ and $\mathcal{R}\left(\Gamma^{\prime}\right)$ are isomorphic and shows at once that topological equivalence is indeed an equivalence relation. According to Remark 1, being in the same MLD-class is not sufficient to guarantee that the tilings are isomorphic. It is sufficient only in case any tile of $\mathcal{T}^{\prime}$ contains exactly one of the punctures of $\mathcal{T}$.

Theorem 6. Two almost-groupoids of finite type tilings are topologically equivalent whenever their associated groupoids are isomorphic.

Proof: We already mentioned above that topological equivalence implies the existence of an isomorphism between the associated groupoids. For the converse let $f$ : $\mathcal{R}\left(\mathcal{M}_{\mathbb{I}}\right) \rightarrow \mathcal{R}\left(\mathcal{M}_{\mathrm{II}}^{\prime}\right)$ be an isomorphism, $\mathcal{R}\left(\mathcal{M}_{\mathbb{I}}\right)=\mathcal{R}\left(\mathcal{M}_{\mathbb{I}}(\mathcal{T})\right), \mathcal{R}\left(\mathcal{M}_{\mathrm{II}}^{\prime}\right)=\mathcal{R}\left(\mathcal{M}_{\mathbb{I I}}\left(\mathcal{T}^{\prime}\right)\right)$. Let $Y \subset \mathcal{R}\left(\mathcal{M}_{\mathbb{I}}\right)$ resp. $Y^{\prime} \subset \mathcal{R}\left(\mathcal{M}_{\mathrm{II}}^{\prime}\right)$ be the set of elements $y$ such that $M_{0}(y)$ is connected. Furthermore, let $X=Y \cup f^{-1}\left(Y^{\prime}\right)$ and $\mathcal{C}(r)=\left\{M_{r}(\alpha) \mid \alpha \in X\right\}$. Since $f: \mathcal{R}\left(\mathcal{M}_{\mathbb{I I}}\right) \rightarrow \mathcal{R}\left(\mathcal{M}_{\mathrm{II}}^{\prime}\right)$ is continuous and $X$ compact,

$$
\forall r^{\prime}>0 \exists r>0 \forall \alpha \in X: f\left(\mathcal{U}_{M_{r}(\alpha)}\right) \subset \mathcal{U}_{M_{r^{\prime}}(f(\alpha))} .
$$

Choose $r>0$ and $r^{\prime}>0$ satisfying (20), and define $\hat{\varphi}: \mathcal{C}(r) \rightarrow \mathcal{M}_{\mathrm{II}}^{\prime}$ by

$$
\hat{\varphi}\left(M_{r}(\alpha)\right):=M_{r^{\prime}}(f(\alpha)) \text {. }
$$

In particular (20) implies

$$
f\left(\mathcal{U}_{c}\right) \subset \mathcal{U}_{\hat{\varphi}(c)}
$$

for all $c \in \mathcal{C}(r)$. To show that $\hat{\varphi}$ is a local derivation rule we first check E1-3. E1 is clearly satisfied. Using set multiplication and the convention that $\mathcal{U}_{c c^{\prime}}=\mathcal{U}_{0}=\emptyset$ if $c \nvdash c^{\prime}$ we obtain for collatable $c_{1}, \ldots, c_{n}$

$$
f\left(\mathcal{U}_{c_{1} \ldots c_{n}}\right)=f\left(\mathcal{U}_{c_{1}}\right) \ldots f\left(\mathcal{U}_{c_{n}}\right) \subset \mathcal{U}_{\varphi\left(c_{1}\right) \ldots \varphi\left(x_{n}\right)}
$$

where we used (9) and that $f$ is a homomorphism of groupoids. Therefore $\mathcal{U}_{\varphi\left(c_{1}\right) \ldots \varphi\left(x_{n}\right)}$ cannot be empty and hence $\varphi$ satisfies E2. To show E3 let $c_{1} \ldots c_{n}$ be a nonzero unit. Then $f\left(\mathcal{U}_{c_{1} \ldots c_{n}}\right) \subset \mathcal{R}\left(\mathcal{M}_{\mathbb{I}}\right)^{0}$. Since, for tilings, either $\mathcal{U}_{c} \cap \mathcal{R}\left(\mathcal{M}_{\mathbb{I}}\right)^{0}=\emptyset$ or $\mathcal{U}_{c} \subset \mathcal{R}\left(\mathcal{M}_{\mathbb{I}}\right)^{0}$ (23) implies E3 for $\varphi$. Therefore $\varphi$ extends to a prehomomorphism. Clearly $D(\varphi)=$ $I(\langle\mathcal{C}\rangle) \preceq \mathcal{M}_{\mathbb{I}}$. Moreover, (23) implies that (22) holds even for all $c \in I(\langle\mathcal{C}\rangle)$. Therefore, if $\left(c_{n}\right)_{n}$ is an approximating sequence, then $f\left(\left[\left(c_{n}\right)_{n}\right]\right) \in \bigcap_{n} \mathcal{U}_{\varphi\left(c_{n}\right)}$ or, stated differently, $f\left(\left[\left(c_{n}\right)_{n}\right]\right) \preceq \widetilde{\varphi}\left(\left[\left(c_{n}\right)_{n}\right]\right)$. Hence if $\varphi$ is approximating then $\mathcal{R}(\varphi)=f$.

So far we have only used that $f$ is a homomorphism. To show that $\varphi$ is approximating we need to use its invertibility. Having nothing specific said about the choice of $r, r^{\prime}$ we choose them now in a way that there exist $0<r_{2} \leq r$ and $r_{1}^{\prime} \geq r^{\prime}$ such that apart from (20) also holds $f^{-1}\left(\mathcal{U}_{M_{r_{1}^{\prime}}(\beta)}\right) \subset \mathcal{U}_{M_{r}\left(f^{-1}(\beta)\right)}$ and $f^{-1}\left(\mathcal{U}_{M_{r^{\prime}}(\beta)}\right) \subset \mathcal{U}_{M_{r_{2}}\left(f^{-1}(\beta)\right)}$ 
for all $\beta \in f(X)$. Since $f(X)$ is compact as well this is possible. We then define $\mathcal{C}^{\prime}(r):=\left\{M_{r}(\beta) \mid \beta \in f(X)\right\}$, and $\hat{\psi}_{1}: \mathcal{C}^{\prime}\left(r_{1}^{\prime}\right) \rightarrow \Gamma, \hat{\psi}_{2}: \mathcal{C}^{\prime}\left(r^{\prime}\right) \rightarrow \Gamma$ by

$$
\hat{\psi}_{1}\left(M_{r_{1}^{\prime}}(\beta)\right):=M_{r}\left(f^{-1}(\beta)\right), \quad \hat{\psi}_{2}\left(M_{r^{\prime}}(\beta)\right):=M_{r_{2}}\left(f^{-1}(\beta)\right)
$$

for all $\beta \in f(X)$. Alike $\varphi, \psi_{i}, i=1,2$, extend to a prehomomorphisms and $\mathcal{R}\left(D\left(\psi_{i}\right)\right)=$ $\mathcal{R}\left(\mathcal{M}_{\mathrm{II}}^{\prime}\right)$. Moreover, $\hat{\varphi} \circ \hat{\psi}_{1}\left(M_{r_{1}^{\prime}}(\beta)\right)=M_{r^{\prime}}(\beta)$ and $\hat{\psi}_{2} \circ \hat{\varphi}\left(M_{r}(\alpha)\right)=M_{r_{2}}(\alpha)$ imply that $\psi_{2} \circ \varphi(c) \succeq c$ for all $c \in D\left(\psi_{2} \circ \varphi\right)$ and $\varphi \circ \psi_{1}\left(c^{\prime}\right) \succeq c^{\prime}$ for all $c^{\prime} \in D\left(\varphi \circ \psi_{1}\right)$. In particular, $\mathcal{R}\left(\varphi \circ \psi_{1}\right)=$ id on $\mathcal{R}\left(\mathcal{M}_{\mathrm{II}}^{\prime}\right)$ and $\mathcal{R}\left(\psi_{2} \circ \varphi\right)=$ id on $\mathcal{R}\left(\mathcal{M}_{\mathbb{I}}\right)$. Therefore, if $\left(c_{n}\right)_{n}$ is an approximating sequence then $\left.\widetilde{\psi}_{2}\left(\left[\left(c_{n}\right)_{n}\right]\right)=\left[\left(\psi_{2} \circ \varphi \circ \psi_{1}\left(c_{n}\right)\right)_{n}\right] \succeq\left[\psi_{1}\left(c_{n}\right)\right)_{n}\right]$. In particular, if next to $\widetilde{c}$ also $\widetilde{\psi}_{2}(\widetilde{c})$ is minimal then $\widetilde{\psi}_{2}(\widetilde{c})=\widetilde{\psi}_{1}(\widetilde{c})$. Now let $\widetilde{c} \in \mathcal{R}\left(\mathcal{M}_{\mathbb{I}}\right)$.

By Lemma 10 there is a $\widetilde{c} \in \mathcal{R}\left(\mathcal{M}_{\mathrm{II}}^{\prime}\right)$ with $\widetilde{c} \preceq \widetilde{\varphi}(\widetilde{c})$. Then $\widetilde{\psi}_{2}\left(\widetilde{c}^{\prime}\right) \preceq \widetilde{\psi}_{2} \circ \widetilde{\varphi}(\widetilde{c})=\widetilde{c}$, i.e. $\widetilde{\psi}_{2}(\widetilde{c})$ is minimal, and hence $\widetilde{c}=\widetilde{\psi}_{1}\left(\widetilde{c}^{\prime}\right)$, and consequently $\widetilde{\varphi}(\widetilde{c})=\widetilde{c}$. It follows that $\widetilde{\varphi}$ is approximating and hence $\mathcal{R}(\varphi)=f$. But then the above implies that $\psi_{i}$, $i=1,2$, are approximating and $\mathcal{R}\left(\psi_{i}\right)=f^{-1}$. Hence $\hat{\varphi}$ and $\hat{\psi}_{i}$ for either of the $i=1,2$ satisfy according to Lemma 2 the requirements of the definition of locally topological equivalence.

q.e.d.

In fact, we have proven a little more, namely that any isomorphism between groupoids associated to finite type tilings is "locally defined", i.e. it can be obtained by a local derivation rule. One could also define a stronger form of topological equivalence between two tilings $\mathcal{T}, \mathcal{T}^{\prime}$ in that one requires in addition for the local morphism of Definition 8 that $\mathcal{R}(\varphi)$ maps the class of $\mathcal{T}$ onto that of $\mathcal{T}^{\prime}$. This is then equivalent to the existence of an isomorphism between $\mathcal{R}\left(\mathcal{M}_{\mathbb{I}}(\mathcal{T})\right)$ and $\mathcal{R}\left(\mathcal{M}_{\mathbb{I}}\left(\mathcal{T}^{\prime}\right)\right)$ which maps the class of $\mathcal{T}$ onto that of $\mathcal{T}^{\prime}$.

A simple example for which the construction of a prehomomorphism of the theorem can be carried out, not yielding an approximating one, is the constant map $f: \mathcal{R}\left(\mathcal{M}_{\mathrm{II}}\right) \rightarrow \mathcal{R}\left(\mathcal{M}_{\mathrm{II}}^{\prime}\right)$ given by $f(\widetilde{c})=\widetilde{u}, \widetilde{u} \in \mathcal{R}\left(\mathcal{M}_{\mathrm{II}}^{\prime}\right)^{0}$ fixed. The above construction yields $\varphi(c)=M_{r^{\prime}}(\widetilde{u})$ for all $c \in D(\varphi)$ which is not approximating.

\section{A SELECTED OVERVIEW ON TOPOLOGICAL INVARIANTS OF TILINGS}

We have shown that the topological groupoid $\mathcal{R}\left(\mathcal{M}_{\mathbb{I}}\right)$ is a complete invariant for a topological equivalence class of tilings which are of finite type. This answers the question under which circumstances two tilings of finite type lead to the same groupoid. Furthermore it means that the groupoid contains all physically interesting topological information about a tiling, the prime example of that being the $K$-theoretic gap labelling.

The question immediately following such a result is that after an invariant for tilinggroupoids which is computable and distinguishes between non-isomorphic groupoids (the term invariant always referring to a quantity which depends on isomorphism classes). In fact, the determination whether two such groupoids are isomorphic or not can be rather difficult, and what we have in mind here is something like Elliot's classification of $A F$-algebras by means of their scaled ordered $K_{0}$-group [Ell76]. These groups may be in many cases easily computed [Eff81. So one might hope that the $K$-theory of the groupoid- $C^{*}$-algebra is a good starting point to classify all groupoids 
coming from tilings. And in fact, if one restricts its attention only to the groupoid$C^{*}$-algebra of the groupoid, then, for 1-dimensional tilings - which may be viewed as topological dynamical systems - one obtains a $C^{*}$-algebra which is the limit of circle algebras. Elliot's classification extends to such algebras [El193], the scaled ordered $K_{0^{-}}$ group of the groupoid- $C^{*}$-algebra is a complete invariant as well. A full treatment of the one dimensional case including an interpretation in dynamical terms can be found in [HPS92, GPS93]. In higher dimensions, it is not yet clear whether $K$-theory yields complete invariants for the groupoid- $C^{*}$-algebras of tilings but the ordered $K_{0}$-group is still an interesting object to consider, after all it has physical signification in the gaplabelling. However, it should be said that there are non-isomorphic tiling groupoids which give rise to isomorphic $C^{*}$-algebras, so that the $K$-theory of the latter cannot be a complete invariant for tiling groupoids. It is known that groupoids are invariants for pairs of $C^{*}$-algebras, the groupoid- $C^{*}$-algebra and a Cartan subalgebra of it Ren80.

3.1. K-theoretic invariants. The definition of the (reduced or full) groupoid $C^{*}$ algebra of an $r$-discrete groupoid can be found in Ren80 or, in the special context of tilings, in Kel95a, Kel95b]. In the latter case, it may be seen as the $C^{*}$-closure of a representation of the inverse semigroup $\mathcal{M}_{\mathbb{I I}} \cup\{0\}$ by means of partial symmetries of a Hilbert space and coincides with the algebra of observables for particles moving in the tiling. To be more precise, a priori on distinguishes two such closures, obtaining the reduced or the full algebra. But since the (discrete) groupoid of a tiling is the abstract transversal of a transformation group with amenable group, its reduced and full groupoid- $C^{*}$-algebra coincide MRW87, Muh.

The $K$-theoretic invariants of the groupoid- $C^{*}$-algebra $\mathcal{A}_{\mathcal{T}}$ of $\mathcal{R}\left(\mathcal{M}_{\mathbb{I}}(\mathcal{T})\right)$ are topological invariants of the tiling. The results which could be obtained so far are, apart from periodic tilings, all related to tilings which are invariant under a primitive invertible substitution. For one dimensional tilings the $K$-theory is computed in [For94, Hos93]. For higher dimensional tilings the (integer) group of coinvariants (which is actually a cohomology group) together with a natural order could be obtained in [Kel95b. For tilings which allow for a locally defined $\mathbb{Z}^{d}$-action, $d \leq 3$, the group of coinvariants embeds as ordered unital group into the $K_{0}$-group. This is enough to solve the $K$ theoretical gap-labelling for these. Explicit calculations include Penrose tilings [Kel95b] and octagonal tilings [Ke195a]. Further results are obtained in terms of cohomology groups, see below.

But before coming to that let us recall Corollary 2 which has as a consequence that $K_{1}$-groups and ordered $K_{0}$-groups alone (without order unit) are invariants for MLDclasses of tilings. That the order unit may distinguish elements of such a class may be seen from the cases in which $\mathcal{R}(\varphi)$ is injective but not surjective. In particular, any tile of $\mathcal{T}^{\prime}$ contains at most one puncture of a tile of $\mathcal{T}$ but some of them carry none. In this situation one can identify $\mathcal{A}_{\mathcal{T}}$ with a full corner of $\mathcal{A}_{\mathcal{T}}$, and the induced order isomorphism between the ordered $K_{0}$-groups maps the order unit of $K_{0}\left(\mathcal{A}_{\mathcal{T}}\right)$ onto an element which is strictly smaller than the order unit of $K_{0}\left(\mathcal{A}_{\mathcal{T}^{\prime}}\right)$ Kel95b.

3.2. Cohomological invariants. Another topological invariant of a groupoid is its cohomology. If one considers cohomology groups of the discrete groupoid with integer coefficients then, at least for tilings which carry a local $\mathbb{Z}^{d}$-action, unordered $K$-groups are isomorphic to cohomology groups [FH95]. E.g. the non-vanishing cohomology group 
of highest degree, which is the group of coinvariants, is a direct summand of the $K_{0^{-}}$ group. This was taken advantage of already above.

On the other hand Anderson and Putnam showed that unordered $K$-theory of two dimensional substitution tilings is isomorphic to the Czech-cohomology of a certain CW-complex AP95. They computed the latter for a number of tilings including Penrose tilings. In particular they obtained as well the $K_{1}$-group. The route they took is different from the one in [Kel95b, but the actual calculations, as far as they concern the common part of the results, reduce at the end in both cases to the computation of images and kernels of combinatorial matrices, which are almost the same.

Comparing the types of invariants it can be said that cohomology groups give a finer grading than $K$-groups but a priori no order. This is a severe draw back due to the vast possibilities of orders on such groups. In particular, integer valued cohomology is not a complete invariant for tilings either.

Other cohomology groups of groupoids are also of interest for physics. The second cohomology group of a groupoid with coefficients in the circle group provides the twisting elements for the construction of the twisted groupoid- $C^{*}$-algebra [Ren80]. For the simpler case of the group $\mathbb{Z}^{2}$ (which is of course a groupoid) the twisted group- $C^{*}$ algebra is very important. It is an irrational rotation algebra which is the observable e.g. for for particles which move on the lattice $\mathbb{Z}^{2}$ (a periodic tiling) and which are subject to a constant perpendicular magnetic field [Zak64, TKNdN82, Bel86]. The flux through the unit cell (a tile) may be interpreted as the cocycle which yields the twisting element. It would therefore be rather interesting to compute the full second cohomology group with coefficients in the circle group for non periodic tilings.

\section{REFERENCES}

[AG95] F. Axel and D. Gratias. Behond Quasicrystals. Springer, 1995.

[AP95] J.E. Anderson and I.F. Putnam. Topological invariants for substitution tilings and their associated $C^{*}$-algebras. Preprint, 1995.

[BBG92] J. Bellissard, A. Bovier, and J.M. Ghez. Gap labelling theorems for one dimensional discrete Schrödinger operators. Rev. Math. Phys., 4:1-38, 1992.

[Bel86] J. Bellissard. K-Theory of $C^{*}$-Algebras in Solid State Physics. In T.C. Dorlas, N.M. Hugenholtz, and M. Winnik, editors, Statistical Mechanics and Field Theory: Mathematical Aspects. Lect. Notes in Phys. 257:99-156, Springer Verlag, 1986.

[Bel92] J. Bellissard. Gap labelling theorems for Schrödinger's operators. In M. Waldschmidt, P. Moussa, J.M. Luck, and C. Itzykson, editors, From Number Theory to Physics. 538630, Springer Verlag, 1992.

[Bla86] B. Blackadar. K-Theory for Operator Algebras. MSRI Publication 5. Springer Verlag, 1986.

[BS95] M. Baake and M. Schlottmann. Geometric aspects of tilings and equivalence concepts. In C. Janot and R. Mosseri, editors, Proc. 5th Int. Conf. on Quasicrystals, pages 15-21. World Scientific, 1995.

[BSJ91] M. Baake, M. Schlottmann, and P.D. Jarvis. Quasiperiodic tilings with tenfold symmetry and equivalence with respect to local derivability. J. Phys. A, 24:4637-4654, 1991.

[Eff81] E.G. Effros. Dimensions and $C^{*}$-Algebras. Conference Board Math. Sci. 46. Amer. Math. Soc. (Providence R.I.), 1981.

[Ell76] G. Elliot. On the classification of inductive limits of sequences of semisimple finite dimensional algebras. J. Algebra, 38:29-44, 1976.

[El193] G. Elliot. On the classification of $C^{*}$-algebras of real rank zero. J. Reine Angew. Math., 443:179-219, 1993. 
[FH95] A.H. Forrest and J. Hunton. The cohomology and K-theory of commuting homeomorphisms of the cantor set. Edinburgh preprint MS95-024, 1995.

[For94] A.H. Forrest. K-groups associated with substitution minimal systems. To appear in Israel J. Math.

[GPS93] T. Giordano, I.F. Putnam, and C.F. Skau. Topological orbit equivalence and $C^{*}$-crossed products. Trondheim preprint Mathematics No. 11/1993, 1993.

[Hos93] B. Host. Dimension groups of substitution dynamical systems. Marseille, unpublished course notes, 1993.

[HPS92] R.H. Herman, I.F. Putnam, and C.F. Skau. Ordered Bratteli diagrams, dimension groups and topological dynamics. Int. J. Math., 3:827-864, 1992.

[JM95] C. Janot and R. Mosseri. Proc. 5th Int. Conf. on Quasicrystals. World Scientific, 1995.

[Kel95a] J. Kellendonk. Integer groups of coinvariants associated to octagonal tilings. Sfb 288 preprint no. 184, to appear, 1995.

[Kel95b] J. Kellendonk. The local structure of tilings and their integer group of coinvariants. King's College London preprint KCL-TH-95-6, cond-mat 9508010, to appear in Commun. Math. Phys., 1995.

[Kel95c] J. Kellendonk. Non commutative geometry of tilings and gap labelling. Rev. Math. Phys., 7:1133-1180, 1995.

[Kum88] A. Kumjian. On equivariant sheaf cohomology and elementary $C^{*}$-bundles. J. Operator Theory, 20:207-240, 1988.

[MRW87] P.S. Muhly, J.N. Renault, and D.P. Williams. Equivalence and isomorphism for groupoid $C^{*}$-algebras. J. Operator Theory, 17:3-22, 1987.

[Muh] P.S. Muhly. private communication.

[Pat93] A. Paterson. Inverse semigroups, groupoids and a problem of J. Renault. In Algebraic Methods in Operator Theory. 11, Birkäuser, 1993.

[Pat95] A. Paterson. The groupoid- $C^{*}$-algebras of inverse semigroups. Preprint, 1995.

[Pet84] M. Petrich. Inverse Semigroups. Pure and applied mathematics. John Wiley \& Sons, 1984.

[Ren80] J. Renault. A Groupoid approach to $C^{*}$-Algebras. Lect. Notes in Math. 793. SpringerVerlag, 1980.

[SO87] P.J. Steinhardt and S. Ostlund. The Physics of Quasicrystals. World Scientific, 1987.

[SS86] J.E.S. Socolar and P.J. Steinhardt. Quasicrystals II: Unit-cell configurations. Phys. Rev. $B, 34(2): 617-647,1986$.

[TKNdN82] D.J. Thouless, M. Kohomoto, M.P. Nightingale, and M. den Nijs. Quantized Hall conductance in two dimensional periodic potential. Phys. Rev. Lett., 49:405, 1982.

[Zak64] J. Zak. Magnetic translation group. Phys. Rev. A, 134:1602-1611, 1964.

Fachbereich Mathematik, Technische Universität Berlin, Strasse des 17. Juni 136, 10623 Berlin, Germany. E-mail: Kellen@math.tu-Berlin.de 